Article

\title{
Novel Antibacterial Polyglycidols: Relationship between Structure and Properties
}

\author{
Fabian Marquardt, Cornelia Stöcker, Rita Gartzen, Elisabeth Heine, Helmut Keul * \\ and Martin Möller *
}

Institute of Technical and Macromolecular Chemistry, RWTH Aachen University and DWI Leibniz-Institute for Interactive Materials, Forckenbeckstr. 50, D-52056 Aachen, Germany; marquardt@dwi.rwth-aachen.de (F.M.); cornelia.stoecker@rwth-aachen.de (C.S.); gartzen@dwi.rwth-aachen.de (R.G.); heine@dwi.rwth-aachen.de (E.H.)

* Correspondence: keul@dwi.rwth-aachen.de (H.K.); moeller@dwi.rwth-aachen.de (M.M.)

Received: 4 December 2017; Accepted: 18 January 2018; Published: 20 January 2018

\begin{abstract}
Antimicrobial polymers are an attractive alternative to low molecular weight biocides, because they are non-volatile, chemically stable, and can be used as non-releasing additives. Polymers with pendant quaternary ammonium groups and hydrophobic chains exhibit antimicrobial properties due to the electrostatic interaction between polymer and cell wall, and the membrane disruptive capabilities of the hydrophobic moiety. Herein, the synthesis of cationic-hydrophobic polyglycidols with varying structures by post-polymerization modification is presented. The antimicrobial properties of the prepared polyglycidols against E. coli and S. aureus are examined. Polyglycidol with statistically distributed cationic and hydrophobic groups (cationic-hydrophobic balance of 1:1) is compared to (i) polyglycidol with a hydrophilic modification at the cationic functionality; (ii) polyglycidol with both—cationic and hydrophobic groups-at every repeating unit; and (iii) polyglycidol with a cationic-hydrophobic balance of 1:2. A relationship between structure and properties is presented.
\end{abstract}

Keywords: antimicrobial polymers; functional polyethers; structure-property relationship; multifunctional polyglycidol; post-polymerization functionalization

\section{Introduction}

Contamination by microorganisms such as bacteria, fungi, and algae is a key issue in medicine [1], pharmaceutical production [2], water purification systems [3], food packaging [4], and various other fields [5,6]. Artificial materials lack defense against microbial growth, allowing microbes to attach to the surface and form a biofilm [7]. One way to prevent the biofilm formation is the usage of disinfectants to keep the surface sterile. Common disinfectants include low molecular weight substances such as alcohols, aldehydes, quaternary ammonium compounds, silver compounds, peroxygens, and bisphenols [8]. However, these classes of antimicrobial substances need to be applied regularly, leading to the development of resistance in the microbial strains [9]. Another way to prevent microbial growth is the coating of surfaces with antimicrobial substances that either kill microorganisms on contact or repel the attachment of microbes [10]. Nevertheless, the leaching of biocides from those coatings causes the same previously mentioned problems. An attractive alternative to low molecular weight biocides are antimicrobial polymers, because they are non-volatile, chemically stable, and can be used as non-releasing additives [11]. These polymers are prepared either by (co-)polymerization of functionalized monomers or by post-polymerization functionalization [12]. Though, the antimicrobial properties are derived from a variety of functionalities such as biguanides [13,14], benzoate esters and benzaldehydes [15], or poly(acrylic acid) [16], the most common active moieties are based on a combination of quaternary ammonium, pyridinium, or phosphonium groups and hydrophobic functionalities [17-19]. The proposed and commonly accepted mechanism for these types of polymers 
features the electrostatic interaction between the cationic moieties and the negatively charged bacterial cell membrane and the disruption of the cell membrane by the hydrophobic groups, leading to leakage and, subsequently, cell death [20,21]. However, due to the heterogeneity and bacterial strain specificity of the cell wall composition, the efficacy of antimicrobial polymers is species-dependent. The outer part of the cell wall of Gram-positive bacteria is composed of about $90 \%$ peptidoglycan. In Gram-negative bacteria, the peptidoglycan layer accounts for approximately $20 \%$ of the cell envelope, being located between the outer membrane and inner cell membrane. Before reaching the inner cell membrane, polymers interact with the negatively charged components of the outer part of the bacterial cell envelope, e.g., teichoic acids in the thick peptidoglycan layers of Gram-positive bacteria, and phospholipids and lipopolysaccharides in the outer membrane of Gram-negative bacteria [22,23]. The effectiveness of the antimicrobial cationic-hydrophobic polymers is dependent on various factors [24]. One factor is the molecular weight of the polymer. Ikeda et al. synthesized polymethacrylates with pendant biguanide units and various molecular weights and tested the antimicrobial activity against Staphylococcus aureus, reaching an optimal activity at molecular weights between 50 and $100 \mathrm{kDa}$ [25]. Kanazawa et al. showed an increase in biocidal activity of poly[tributyl(4-vinylbenzyl)phosphonium chloride] against $S$. aureus with increasing molecular weight [26]. Based on the described mechanism, they assume that a higher molecular weight and a consequential higher charge density enhance the adsorption of the polymers to the cell membrane. A stronger adsorption leads to a stronger disruption of the cell membrane and, thus, to a higher activity of the polymer. However, Lienkamp et al. found that when the molecular weight reaches a threshold value, the efficacy of the polymers decreases [27]. Additionally, Locock et al. reported the reverse effect on guanylated polymethacrylates [28]. The presented polymers showed higher antimicrobial activity at lower molecular weights, proving that the efficacy of antimicrobial polymers does not exclusively depend on their molecular weight. A second factor for the antimicrobial behavior is the alkyl chain length of the hydrophobic moiety. However, the optimal alkyl chain length is different for different types of polymers. Pasquier et al. prepared branched poly(ethylene imine)s with pendant ammonium and alkyl functionalities, ranging from $C_{6}$ to $C_{16}$. An increase in the alkyl chain length lead to an increase in the antimicrobial activity against $E$. coli [29]. The activity was further enhanced by He et al., attaching the hydrophobic chain directly to the ammonium group [30]. The opposite influence of aliphatic side chains was reported by Xu et al. on comb-like ionenes [31]. Here, a decrease in the alkyl chain length lead to an increase in the antimicrobial activity against $E$. coli. On the other hand, Chen et al. prepared poly(propylene imine) dendrimers with alkyl chains ranging from $C_{8}$ to $\mathrm{C}_{16}$, showing a parabolic dependence between the biocidal effect and the alkyl chain length, with the highest activity at $\mathrm{C}_{10}$ [32].

In the current work, we present the preparation of various antimicrobial polymers based on polyglycidol with quaternary trimethylammonium groups as the cationic moiety and dodecyl chains as the hydrophobic part. Polyglycidol is a highly functional polymer with a hydroxy group in every repeating unit, allowing various further modifications [33,34]. It is non-toxic, soluble in aqueous media, and licensed by the Food and Drug Administration (FDA) [35,36]. The cationic and hydrophobic side chain functionalities were distributed statistically along the polymer backbone (cationic-hydrophobic ratio of 1:1). This functionalized polyglycidol was compared to (i) a polyglycidol modified with hydrophilic hydroxyethyl functionalities at the cationic center; (ii) a polyglycidol with cationic and hydrophobic moieties at every repeating unit; and (iii) a polyglycidol with a cationic-hydrophobic balance of 1:2. All polymers were tested in regard to their antimicrobial properties against Escherichia coli and Staphylococcus aureus to examine a possible relationship between the structure and the biocidal effect of the polymer. 


\section{Materials and Methods}

\subsection{Materials}

Potassium tert-butoxide (1 M solution in THF, Sigma Aldrich Chemie GmbH, Steinheim, Germany), diglyme ( $\geq 99 \%$, extra dry, over molecular sieves, Thermo Fisher Acros Organics, Geel, Belgium), pyridine (99.5\%, extra dry, over molecular sieves, Thermo Fisher Acros Organics, Geel, Belgium), phenyl chloroformate ( $>97 \%$, Sigma Aldrich Chemie GmbH, Steinheim, Germany), 4-nitrophenyl chloroformate (>98\%, TCI Deutschland GmbH, Eschborn, Germany), 3-(dimethylamino)-1-propylamine (99\%, Thermo Fisher Acros Organics, Geel, Belgium), N-(3-aminopropyl)diethanolamine ( $>90 \%$, TCI Deutschland GmbH, Eschborn, Germany), dodecylamine (98\%, Sigma Aldrich Chemie GmbH, Steinheim, Germany), 4-dimethylaminopyridine (>98\%, Sigma Aldrich Chemie GmbH, Steinheim, Germany), DL-homocysteine thiolactone hydrochloride (98\%, abcr GmbH, Karlsruhe, Germany), triethylamine ( $\geq 99.5 \%$, anhydrous, Sigma Aldrich Chemie GmbH, Steinheim, Germany), dodecyl acrylate (>98\%, TCI Deutschland GmbH, Eschborn, Germany), methyl iodide (>99\%, Sigma Aldrich Chemie $\mathrm{GmbH}$, Steinheim, Germany), tetrahydrofuran ( $99.8 \%$, stabilizer free, extra dry, Thermo Fisher Acros Organics, Geel, Belgium), chloroform (99.9\%, extra dry, over molecular sieves, Thermo Fisher Acros Organics, Geel, Belgium), N,N-dimethylformamide (99.8\%, extra dry, over molecular sieves, Thermo Fisher Acros Organics, Geel, Belgium), methanol ( $\geq 99.8 \%$, p.a., Th. Geyer CHEMSOLUTE ${ }^{\circledR}$, Renningen, Germany), and dichloromethane ( $\geq 99.8 \%$, anhydrous, Sigma Aldrich Chemie GmbH, Steinheim, Germany) were used as received.

The 3-phenyl-1-propanol (99\%, Sigma Aldrich Chemie GmbH, Steinheim, Germany) was stirred with calcium hydride for $24 \mathrm{~h}$ and then distilled. Ethoxyethyl glycidyl ether (EEGE) was synthesized from 2,3-epoxypropan-1-ol (glycidol, 96\%, Sigma Aldrich Chemie GmbH, Steinheim, Germany) and ethyl vinyl ether (99\%, Sigma Aldrich Chemie GmbH, Steinheim, Germany) according to Fitton et al. [37], purified by distillation, and stored under a nitrogen atmosphere over a molecular sieve (3 A). Polyglycidol (PG) (1) was synthesized according to literature [38].

Water-sensitive reactions were carried out in a nitrogen atmosphere. Nitrogen (Linde 5.0) was passed over a molecular sieve $(4 \AA)$ and finely distributed potassium on aluminum oxide.

\subsection{Bacteria}

To determine the antibacterial activity, polymers were tested against the Gram-negative bacterium Escherichia coli (DSM498) and the Gram-positive bacterium Staphylococcus aureus (ATCC6538). Overnight cultures of E. coli and S. aureus with defined bacterial count in Mueller-Hinton broth (Sigma Aldrich Chemie $\mathrm{GmbH}$, Steinheim, Germany, $\mathrm{pH}=7.4 \pm 0.2$ ) were used as inoculate in the antibacterial test.

\subsection{Antibacterial Tests of Polymer Solutions}

The antibacterial activity of the polymers in solution was determined by measuring the minimal inhibitory concentration (MIC) using the test bacteria mentioned above. Suspensions of strains with known colony forming units (CFU; $1 \times 10^{6}-2 \times 10^{6} \mathrm{CFU} \cdot \mathrm{mL}^{-1}$ ) were incubated at $37^{\circ} \mathrm{C}$ in nutrient solutions (Mueller-Hinton Broth, $\mathrm{MHB}$ ) with different concentrations of the polymer samples. The polymer samples were solubilized in bidistilled water and added to the nutrient solution at a constant ratio of 1:10. The growth of the bacteria was followed during the incubation over $20 \mathrm{~h}$ by measuring the optical density at $612 \mathrm{~nm}$ every $30 \mathrm{~min}$ (with $1400 \mathrm{~s}$ of shaking at $100 \mathrm{rpm}$ per $30 \mathrm{~min}$ cycle by using a microplate reader/incubator (TECAN Infinite 200 Pro, Tecan Trading AG, Männedorf, Switzerland). The testing is performed with defined concentrations specifically for each polymer until, within the monitoring time of $20 \mathrm{~h}$, no bacterial growth curve is recorded. All experiments were performed in triplicate duplicates and MIC determination was repeated on three different days. The polymers were not sterilized. Sterile controls (defined polymer concentrations in nutrient solution without bacteria) were assessed in every growth curve monitoring testing series. MICs were 
determined according to broth microdilution in 96-well microtitre plates [39]. Antimicrobial polymer testing reference polymers/controls $\varepsilon$-polylysine and polyhexanide (poly(hexamethylene biguanide)) were used.

The minimal inhibitory concentration (MIC) corresponds to the concentration of the test substance at which a complete inhibition of the growth of the inoculated bacteria was observed by comparison with control samples without test substance.

\subsection{Hemolytic Activity}

Hemolytic activity was assessed according to literature [40]. Human erythrocytes (from healthy donors, red blood cells (RBC), 0 , Rh positive, citrate-stabilized) were obtained by centrifugation (3500 rpm, $12 \mathrm{~min}$ ) to remove plasma, washed three times in PBS (0.01 M phosphate buffered saline, Sigma Aldrich Chemie GmbH, Steinheim, Germany), and diluted in PBS to obtain a stock solution of $2.5 \times 10^{8}-3.0 \times 10^{8} / \mathrm{mL}$ RBC. Solutions of defined polymer concentration $(250 \mu \mathrm{L})$ were pipetted into $250 \mu \mathrm{L}$ of the stock solution; the final amount of RBC being $1.2 \times 10^{8}-1.5 \times 10^{8} \mathrm{RBC} / \mathrm{mL}$. The RBC were exposed for $60 \mathrm{~min}$ at $37^{\circ} \mathrm{C}$ under 3D-shaking; centrifuged thereafter (4000 rpm, $12 \mathrm{~min}$ ) and the absorption of the supernatant (diluted 10-fold in PBS) was determined at $414 \mathrm{~nm}$ in a microplate reader. As reference solutions, (i) PBS for determining spontaneous hemolysis and (ii) 1\% Triton X-100 for $100 \%$ hemolysis (positive control) were used. Hemolysis was plotted as a function of polymer concentration and the hemolytic activity was defined as the polymer concentration that causes $50 \%$ hemolysis of human $\mathrm{RBC}$ relative to the positive control $\left(\mathrm{HC}_{50}\right)$.

\subsection{Measurements}

${ }^{1} \mathrm{H}$ NMR and ${ }^{13} \mathrm{C}$ NMR were recorded on a Bruker DPX-400 FT-NMR spectrometer (Bruker Corporation, Billerica, MA, USA) at 400 and $101 \mathrm{MHz}$, respectively. Deuterated chloroform $\left(\mathrm{CDCl}_{3}\right)$, deuterated dimethyl sulfoxide (DMSO- $d_{6}$ ), deuterated acetone (acetone- $d_{6}$ ), and deuterated methanol (MeOD) were used as solvents. The residual solvent signal was used as internal standard. Coupling constants $J_{x y}$ are given in $\mathrm{Hz}$.

Molecular weights $\left(M_{n, \mathrm{SEC}}\right)$ and molecular weight distributions $(\nexists)$ were determined by size exclusion chromatography (SEC). SEC analyses were carried out with DMF as eluent. SEC with DMF (HPLC grade, VWR) as eluent was performed using an Agilent 1100 system (Agilent Technologies, Santa Clara, CA, USA) equipped with a dual RI-/Visco detector (ETA-2020, WGE Dr. Bures $\mathrm{GmbH} \& \mathrm{Co} \mathrm{KG}$, Dallgow-Doeberitz, Germany). The eluent contained $1 \mathrm{~g} \cdot \mathrm{L}^{-1} \mathrm{LiBr}(\geq 99 \%$, Sigma Aldrich Chemie $\mathrm{GmbH}$, Steinheim, Germany). The sample solvent contained traces of distilled water as internal standard. For cationic samples, the eluent contained $2 \mathrm{~g} \cdot \mathrm{L}^{-1} \mathrm{LiBr}$ and $2 \mathrm{~g} \cdot \mathrm{L}^{-1}$ tris(hydroxymethyl)aminomethane (TRIS, ultrapure grade, $\geq 99.9 \%$, Sigma Aldrich Chemie $\mathrm{GmbH}$, Steinheim, Germany). One pre-column $(8 \mathrm{~mm} \times 50 \mathrm{~mm})$ and four GRAM gel columns $(8 \mathrm{~mm} \times 300 \mathrm{~mm}$, Polymer Standards Service, Mainz, Germany) were applied at a flow rate of $1.0 \mathrm{~mL} \cdot \mathrm{min}^{-1}$ at $40^{\circ} \mathrm{C}$. The diameter of the gel particles measured $10 \mu \mathrm{m}$, the nominal pore widths were 30, 100, 1000, and $3000 \AA$. Calibration was achieved using narrowly distributed poly(methyl methacrylate) standards (Polymer Standards Service, Mainz, Germany). Results were evaluated using the PSS WinGPC UniChrom software (Version 8.1.1, PSS Polymer Standards Service GmbH, Mainz, Germany).

Dialysis was performed in methanol using Biotech CE Tubing (MWCO: 100-500 D, $3.1 \mathrm{~mL} \cdot \mathrm{cm}^{-1}$, Spectrum Laboratories, Inc., Rancho Dominguez, CA, USA) and Biotech RC Tubing (MWCO: 1 kD, $6.4 \mathrm{~mL} \cdot \mathrm{cm}^{-1}$, Spectrum Laboratories, Inc., Rancho Dominguez, CA, USA), respectively. The membrane was washed for $15 \mathrm{~min}$ in water before use to remove the sodium azide solution.

\subsection{Synthesis of Poly (glycidyl phenyl carbonate) $\left(P\left(G^{P C}\right)_{27}\right)(2)$}

$\mathrm{PG}_{27}$ (1) $(2.018 \mathrm{~g}, 27.24 \mathrm{mmol} \mathrm{OH})$ was dissolved in pyridine $(18.87 \mathrm{~mL})$, and a solution of phenyl chloroformate $(4.692 \mathrm{~g}, 29.97 \mathrm{mmol})$ in dichloromethane $(17.5 \mathrm{~mL})$ was added in $30 \mathrm{~min}$ 
at $0{ }^{\circ} \mathrm{C}$ using a syringe pump. The reaction mixture was allowed to warm to room temperature and stirred for $20 \mathrm{~h}$. The precipitate was removed by filtration. The solution was washed with water $(15 \mathrm{~mL}), 1 \mathrm{M} \mathrm{HCl}$ solution (aq.) $(3 \times 15 \mathrm{~mL})$, and saturated $\mathrm{NaCl}$ solution (aq.) $(15 \mathrm{~mL})$. The organic phase was dried over $\mathrm{Na}_{2} \mathrm{SO}_{4}$, filtrated, and the solvent removed under reduced pressure. Polymer 2 was obtained as a brown viscous liquid $(3.650 \mathrm{~g}, 69 \%) \cdot M_{\mathrm{n}, \mathrm{NMR}}=5243 \mathrm{~g} \cdot \mathrm{mol}^{-1}$, $M_{n, S E C}=4800 \mathrm{~g} \cdot \mathrm{mol}^{-1}, \oplus=1.14 .{ }^{1} \mathrm{H} \mathrm{NMR}\left(400 \mathrm{MHz}, \mathrm{CDCl}_{3}\right)(2): \delta=1.79\left(\mathrm{~m}, \mathrm{ArCH}_{2} \mathrm{CH}_{2}\right)$, $2.57\left(\mathrm{t},{ }^{3} \mathrm{~J}_{\mathrm{HH}}=7.8 \mathrm{~Hz}, \mathrm{ArCH}_{2} \mathrm{CH}_{2}\right), 3.45-3.87\left(\mathrm{~m}, \mathrm{ArCH}_{2} \mathrm{CH}_{2} \mathrm{CH}_{2}, \mathrm{OCH}_{2} \mathrm{CH}\left(\mathrm{CH}_{2} \mathrm{OC}=\mathrm{OOPh}\right) \mathrm{O}\right)$, 4.08-4.42 (m, $\left.\mathrm{OCH}_{2} \mathrm{CH}\left(\mathrm{CH}_{2} \mathrm{OC}=\mathrm{OOPh}\right) \mathrm{O}\right), 6.98-7.29\left(\mathrm{~m}, \mathrm{ArCH}_{2} \mathrm{CH}_{2},(\mathrm{OC}=\mathrm{OOPh}) \mathrm{O}\right) \mathrm{ppm} .{ }^{13} \mathrm{C} \mathrm{NMR}$ $\left(101 \mathrm{MHz}, \mathrm{CDCl}_{3}\right)(2): \delta=31.2\left(\mathrm{ArCH}_{2} \mathrm{CH}_{2}\right), 32.3\left(\mathrm{ArCH}_{2} \mathrm{CH}_{2}\right), 67.7-69.4\left(\mathrm{ArCH}_{2} \mathrm{CH}_{2} \mathrm{CH}_{2}\right.$, $\left.\left.\mathrm{OCH}_{2} \mathrm{CH}\left(\mathrm{CH}_{2} \mathrm{OC}=\mathrm{OOPh}\right) \mathrm{O}\right), 77.4\left(\mathrm{OCH}_{2} \mathrm{CH}\left(\mathrm{CH}_{2} \mathrm{OC}=\mathrm{OOPh}\right) \mathrm{O}\right), 121.0(\mathrm{OC}=\mathrm{OOPh}) \mathrm{O}\right), 126.1\left(\mathrm{ArCH} \mathrm{CH}_{2}\right.$, $\left.\left.\mathrm{OC}=\mathrm{OOPh}) \mathrm{O}), 128.4\left(\mathrm{ArCH}_{2}\right), 128.5\left(\mathrm{ArCH}_{2}\right), 129.6(\mathrm{OC}=\mathrm{OOPh}) \mathrm{O}\right), 141.8\left(\operatorname{ArCH}_{2}\right), 151.1(\mathrm{OC}=\mathrm{OOPh}) \mathrm{O}\right)$, $153.6(\mathrm{OC}=\mathrm{OOPh}) \mathrm{O}) \mathrm{ppm}$.

2.7. Synthesis of Poly(glycidyl 3-dimethylaminopropylcarbamate-co-glycidyl dodecylcarbamate)

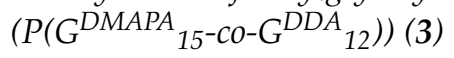

$\mathrm{P}\left(\mathrm{G}^{\mathrm{PC}}\right)_{27}$ (2) (1.509 $\mathrm{g}, 7.77 \mathrm{mmol}$ carbonate) was dissolved in tetrahydrofuran $(15 \mathrm{~mL})$ and a solution of 3-(dimethylamino)-1-propylamine (0.397 $\mathrm{g}, 3.89 \mathrm{mmol}$ ) and dodecylamine (0.721 g, $3.89 \mathrm{mmol})$ in tetrahydrofuran $(15 \mathrm{~mL})$ was added in $1 \mathrm{~h} \mathrm{at} 0{ }^{\circ} \mathrm{C}$ using a syringe pump. The reaction was allowed to warm to room temperature and stirred for $42 \mathrm{~h}$. The solvent was removed under reduced pressure and the polymer purified by dialysis in methanol. Polymer 3 was obtained as a yellowish viscous liquid $(1.153 \mathrm{~g}, 62 \%)$. $M_{n, \mathrm{NMR}}=6459 \mathrm{~g} \cdot \mathrm{mol}^{-1}, M_{\mathrm{n}, \mathrm{SEC}}=7600 \mathrm{~g} \cdot \mathrm{mol}^{-1}, \Xi=1.38 .{ }^{1} \mathrm{H}$ NMR $\left(400 \mathrm{MHz}, \mathrm{CDCl}_{3}\right)$ (3): $\delta=0.84\left(\mathrm{t},{ }^{3} J_{\mathrm{HH}}=7.0 \mathrm{~Hz}, \mathrm{NHCH}_{2} \mathrm{CH}_{2}\left(\mathrm{CH}_{2}\right)_{9} \mathrm{CH}_{3}\right), 1.21\left(\mathrm{~s}, \mathrm{NHCH}_{2} \mathrm{CH}_{2}\left(\mathrm{CH}_{2}\right)_{9} \mathrm{CH}_{3}\right)$, 1.34-1.51 (m, $\left.\mathrm{NHCH}_{2} \mathrm{CH}_{2}\left(\mathrm{CH}_{2}\right)_{9} \mathrm{CH}_{3}\right), 1.54-1.68\left(\mathrm{~m}, \mathrm{NHCH}_{2} \mathrm{CH}_{2} \mathrm{CH}_{2} \mathrm{~N}\left(\mathrm{CH}_{3}\right)_{2}\right), 1.77-1.89$ (m,

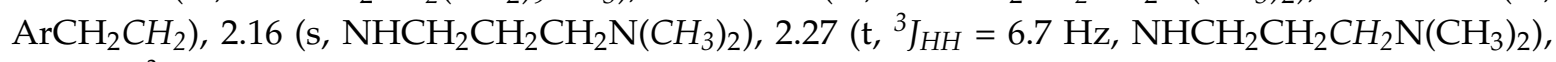
$2.63\left(\mathrm{t},{ }^{3} J_{\mathrm{HH}}=7.6 \mathrm{~Hz}, \mathrm{ArCH}_{2} \mathrm{CH}_{2}\right), 3.01-3.11\left(\mathrm{~m}, \mathrm{NHCH}_{2} \mathrm{CH}_{2}\left(\mathrm{CH}_{2}\right)_{9} \mathrm{CH}_{3}\right), 3.12-3.21(\mathrm{~m}, 2 \mathrm{H}$, $\left.\mathrm{NHCH}_{2} \mathrm{CH}_{2} \mathrm{CH}_{2} \mathrm{~N}\left(\mathrm{CH}_{3}\right)_{2}\right), 3.45-3.79\left(\mathrm{~m}, \mathrm{ArCH}_{2} \mathrm{CH}_{2} \mathrm{CH}_{2}, \mathrm{OCH}_{2} \mathrm{CH}\left(\mathrm{CH}_{2} \mathrm{OC}=\mathrm{ONHR}\right) \mathrm{O}\right), 3.86-4.36$ (m, $\left.\mathrm{OCH}_{2} \mathrm{CH}\left(\mathrm{CH}_{2} \mathrm{OC}=\mathrm{ONHR}\right) \mathrm{O}\right), 5.88$ (br. s, NH), 6.17 (br. s, NH), 7.09-7.25 (m, $A r \mathrm{CH}_{2} \mathrm{CH}_{2}$ ) ppm. ${ }^{13} \mathrm{C} \mathrm{NMR}\left(101 \mathrm{MHz}, \mathrm{CDCl}_{3}\right)(3): \delta=14.2\left(\mathrm{NHCH}_{2} \mathrm{CH}_{2}\left(\mathrm{CH}_{2}\right)_{9} \mathrm{CH}_{3}\right), 22.7\left(\mathrm{NHCH}_{2}\left(\mathrm{CH}_{2}\right)_{10} \mathrm{CH}_{3}\right), 27.0$ $\left(\mathrm{NHCH}_{2} \mathrm{CH}_{2} \mathrm{CH}_{2} \mathrm{~N}\left(\mathrm{CH}_{3}\right)_{2}, 27.5-32.0\left(\mathrm{NHCH}_{2}\left(\mathrm{CH}_{2}\right)_{10} \mathrm{CH}_{3}, \mathrm{ArCH}_{2} \mathrm{CH}_{2}\right), 39.9\left(\mathrm{NHCH}_{2}\left(\mathrm{CH}_{2}\right)_{10} \mathrm{CH}_{3}\right)\right.$, $41.2\left(\mathrm{NHCH}_{2} \mathrm{CH}_{2} \mathrm{CH}_{2} \mathrm{~N}\left(\mathrm{CH}_{3}\right)_{2}\right), 45.5\left(\mathrm{NHCH}_{2} \mathrm{CH}_{2} \mathrm{CH}_{2} \mathrm{~N}\left(\mathrm{CH}_{3}\right)_{2}\right)$, $57.6\left(\mathrm{NHCH}_{2} \mathrm{CH}_{2} \mathrm{CH}_{2} \mathrm{~N}\left(\mathrm{CH}_{3}\right)_{2}\right)$, 64.2-70.0 ( $\left.\mathrm{ArCH}_{2} \mathrm{CH}_{2} \mathrm{CH}_{2}, \mathrm{OCH}_{2} \mathrm{CH}\left(\mathrm{CH}_{2} \mathrm{OC}=\mathrm{ONHR}\right) \mathrm{O}\right), 78.0\left(\mathrm{OCH}_{2} \mathrm{CH}\left(\mathrm{CH}_{2} \mathrm{OC}=\mathrm{ONHR}\right) \mathrm{O}\right), 125.8$ $\left(\mathrm{ArCH} \mathrm{CH}_{2}\right), 128.4\left(\mathrm{ArCH} \mathrm{CH}_{2}\right), 128.5\left(\mathrm{ArCH} \mathrm{CH}_{2}\right), 141.9\left(\mathrm{ArCH}_{2}\right), 156.7\left(\mathrm{OCH}_{2} \mathrm{CH}\left(\mathrm{CH}_{2} \mathrm{OC}=\mathrm{ONHR}\right) \mathrm{O}\right) \mathrm{ppm}$.

\subsection{Synthesis of Poly(glycidyl 3-aminopropyldiethanolcarbamate-co-glycidyl dodecylcarbamate)} $\left(P\left(G^{A P D E A}{ }_{16}-C O-G^{D D A}{ }_{11}\right)\right)(4)$

$\mathrm{P}\left(\mathrm{G}^{\mathrm{PC}}\right)_{27}(2)(0.707 \mathrm{~g}, 3.51 \mathrm{mmol}$ carbonate $)$ was dissolved in tetrahydrofuran $(10 \mathrm{~mL})$ and a solution of $\mathrm{N}$-(3-aminopropyl)diethanolamine $(0.284 \mathrm{~g}, 1.75 \mathrm{mmol})$, and dodecylamine $(0.325 \mathrm{~g}, 1.75 \mathrm{mmol})$ in tetrahydrofuran $(5 \mathrm{~mL})$ was added in $1 \mathrm{~h}$ at $0{ }^{\circ} \mathrm{C}$ using a syringe pump. The reaction was allowed to warm to room temperature and stirred for $42 \mathrm{~h}$. The solvent was removed under reduced pressure and the polymer purified by dialysis in methanol. Polymer 4 was obtained as a colorless viscous liquid $(0.655 \mathrm{~g}, 66 \%) \cdot M_{\mathrm{n}, \mathrm{NMR}}=7337 \mathrm{~g} \cdot \mathrm{mol}^{-1}, M_{\mathrm{n}, \mathrm{SEC}}=14,100 \mathrm{~g} \cdot \mathrm{mol}^{-1}, Ð=3.56 .{ }^{1} \mathrm{H} \mathrm{NMR}(400 \mathrm{MHz}$, MeOD) (4): $\delta=0.92\left(\mathrm{t},{ }^{3} \mathrm{JHH}_{\mathrm{HH}}=7.0 \mathrm{~Hz}, \mathrm{NHCH}_{2} \mathrm{CH}_{2}\left(\mathrm{CH}_{2}\right)_{9} \mathrm{CH}_{3}\right), 1.31\left(\mathrm{~s}, \mathrm{NHCH}_{2} \mathrm{CH}_{2}\left(\mathrm{CH}_{2}\right)_{9} \mathrm{CH}_{3}\right)$, 1.44-1.59 (m, $\left.\mathrm{NHCH}_{2} \mathrm{CH}_{2}\left(\mathrm{CH}_{2}\right)_{9} \mathrm{CH}_{3}\right), 1.62-1.76\left(\mathrm{~m}, \mathrm{NHCH}_{2} \mathrm{CH}_{2} \mathrm{CH}_{2} \mathrm{~N}\left(\mathrm{CH}_{2} \mathrm{CH}_{2} \mathrm{OH}\right)_{2}\right), 1.85-1.92(\mathrm{~m}$, $\left.\mathrm{ArCH}_{2} \mathrm{CH}_{2}\right), 2.55-2.74\left(\mathrm{~m}, \mathrm{CH}_{2} \mathrm{~N}\left(\mathrm{CH}_{2} \mathrm{CH}_{2} \mathrm{OH}\right)_{2}\right), 3.06-3.14\left(\mathrm{~m}, \mathrm{NHCH}_{2} \mathrm{CH}_{2}\left(\mathrm{CH}_{2}\right)_{9} \mathrm{CH}_{3}\right), 3.15-3.23$ $\left(\mathrm{m}, \mathrm{NHCH}_{2} \mathrm{CH}_{2} \mathrm{CH}_{2} \mathrm{~N}\left(\mathrm{CH}_{2} \mathrm{CH}_{2} \mathrm{OH}\right)_{2}\right), 3.62\left(\mathrm{t},{ }^{3} \mathrm{~J}_{\mathrm{HH}}=5.4 \mathrm{~Hz}, \mathrm{CH}_{2} \mathrm{~N}\left(\mathrm{CH}_{2} \mathrm{CH}_{2} \mathrm{OH}\right)_{2}\right), 3.66-3.81(\mathrm{~m}$, $\left.\mathrm{ArCH}_{2} \mathrm{CH}_{2} \mathrm{CH}_{2}, \mathrm{OCH}_{2} \mathrm{CH}\left(\mathrm{CH}_{2} \mathrm{OC}=\mathrm{ONHR}\right) \mathrm{O}\right), 4.00-4.34$ (m, $\left.\mathrm{OCH}_{2} \mathrm{CH}\left(\mathrm{CH}_{2} \mathrm{OC}=\mathrm{ONHR}\right) \mathrm{O}\right), 7.17-7.32$ (m, $\left.\mathrm{ArCH}_{2} \mathrm{CH}_{2}\right)$ ppm. ${ }^{13} \mathrm{C} \mathrm{NMR}(101 \mathrm{MHz}, \mathrm{MeOD})(4): \delta=14.6\left(\mathrm{NHCH}_{2} \mathrm{CH}_{2}\left(\mathrm{CH}_{2}\right)_{9} \mathrm{CH}_{3}\right), 23.8-30.9$ $\left(\mathrm{NHCH}_{2} \mathrm{CH}_{2}\left(\mathrm{CH}_{2}\right)_{9} \mathrm{CH}_{3}\right), 28.2\left(\mathrm{NHCH}_{2} \mathrm{CH}_{2} \mathrm{CH}_{2} \mathrm{~N}\left(\mathrm{CH}_{2} \mathrm{CH}_{2} \mathrm{OH}\right)_{2}\right), 33.1\left(\mathrm{NHCH}_{2} \mathrm{CH}_{2}\left(\mathrm{CH}_{2}\right)_{9} \mathrm{CH}_{3}\right), 40.1$ $\left(\mathrm{NHCH}_{2} \mathrm{CH}_{2} \mathrm{CH}_{2} \mathrm{~N}\left(\mathrm{CH}_{2} \mathrm{CH}_{2} \mathrm{OH}\right)_{2}\right)$, $42.0\left(\mathrm{NHCH}_{2} \mathrm{CH}_{2}\left(\mathrm{CH}_{2}\right)_{9} \mathrm{CH}_{3}\right)$, $53.6\left(\mathrm{CH}_{2} \mathrm{~N}\left(\mathrm{CH}_{2} \mathrm{CH}_{2} \mathrm{OH}\right)_{2}\right)$, 
$57.6\left(\mathrm{CH}_{2} \mathrm{~N}\left(\mathrm{CH}_{2} \mathrm{CH}_{2} \mathrm{OH}\right)_{2}\right), \quad 60.8 \quad\left(\mathrm{CH}_{2} \mathrm{~N}\left(\mathrm{CH}_{2} \mathrm{CH}_{2} \mathrm{OH}\right)_{2}\right), \quad 65.3-79.3 \quad\left(\mathrm{ArCH}_{2} \mathrm{CH}_{2} \mathrm{CH}_{2}\right.$, $\left.\mathrm{OCH}_{2} \mathrm{CH}\left(\mathrm{CH}_{2} \mathrm{OC}=\mathrm{ONHR}\right) \mathrm{O}\right), 129.4\left(\mathrm{ArCH}_{2}\right), 129.6\left(\mathrm{ArCH}_{2}\right), 158.7\left(\mathrm{OCH}_{2} \mathrm{CH}\left(\mathrm{CH}_{2} \mathrm{OC}=\mathrm{ONHR}\right) \mathrm{O}\right) \mathrm{ppm}$.

2.9. Synthesis of Poly(glycidyl 3-trimethylaminopropylcarbamate-co-glycidyl dodecylcarbamate) $\left(P\left(G^{T M A P A}{ }_{15}-C O-G^{D D A}{ }_{12}\right)\right)(5)$

$\mathrm{P}\left(\mathrm{G}^{\mathrm{DMAPA}}{ }_{15}-\mathrm{co}-\mathrm{G}^{\mathrm{DDA}}{ }_{12}\right)$ (3) $\left(0.147 \mathrm{~g}, 0.34 \mathrm{mmol}-\mathrm{NMe}_{2}\right)$ was dissolved in THF $(3.0 \mathrm{~mL})$. Methyl iodide $(0.058 \mathrm{~g}, 0.41 \mathrm{mmol})$ was added and the solution was stirred for $20 \mathrm{~h}$ at room temperature. Excess methyl iodide and the solvent were removed under reduced pressure and the polymer purified by dialysis in methanol. Polymer $\mathbf{5}$ was obtained as a yellowish crystalline solid $(0.205 \mathrm{~g}, 89 \%) . \quad M_{n, \mathrm{NMR}}=10,111 \mathrm{~g} \cdot \mathrm{mol}^{-1}, M_{n, \mathrm{SEC}}=4400 \mathrm{~g} \cdot \mathrm{mol}^{-1}$, $Ð=$ 1.18. ${ }^{1} \mathrm{H}$ NMR $\left(400 \mathrm{MHz}, \mathrm{DMSO}-d_{6}\right)(5): \delta=0.74-0.90\left(\mathrm{~m}, \mathrm{NHCH}_{2} \mathrm{CH}_{2}\left(\mathrm{CH}_{2}\right)_{9} \mathrm{CH}_{3}\right)$, 1.20 (s, $\left.\quad \mathrm{NHCH}_{2} \mathrm{CH}_{2}\left(\mathrm{CH}_{2}\right)_{9} \mathrm{CH}_{3}\right), \quad 1.30-1.44$ (m, $\left.\mathrm{NHCH}_{2} \mathrm{CH}_{2}\left(\mathrm{CH}_{2}\right)_{9} \mathrm{CH}_{3}\right), \quad 1.72-1.91$ (m, $\left.\mathrm{NHCH}_{2} \mathrm{CH}_{2} \mathrm{CH}_{2} \mathrm{~N}^{+}\left(\mathrm{CH}_{3}\right)_{3}, \mathrm{ArCH}_{2} \mathrm{CH}_{2}\right), 2.55-2.62\left(\mathrm{~m},{ }^{3} J_{\mathrm{HH}}=7.6 \mathrm{~Hz}, \mathrm{ArCH}_{2} \mathrm{CH}_{2}\right), 2.85-2.99$ (m, $\left.\mathrm{NHCH}_{2} \mathrm{CH}_{2}\left(\mathrm{CH}_{2}\right)_{9} \mathrm{CH}_{3}\right), 3.00-3.17\left(\mathrm{~m}, \mathrm{NHCH}_{2} \mathrm{CH}_{2} \mathrm{CH}_{2} \mathrm{~N}^{+}\left(\mathrm{CH}_{3}\right)_{3}, \mathrm{NHCH}_{2} \mathrm{CH}_{2} \mathrm{CH}_{2} \mathrm{~N}^{+}\left(\mathrm{CH}_{3}\right)_{3}\right)$, 3.26-3.43 (m, $\left.\mathrm{NHCH}_{2} \mathrm{CH}_{2} \mathrm{CH}_{2} \mathrm{~N}^{+}\left(\mathrm{CH}_{3}\right)_{3}\right)$, 3.44-3.74 (m, $\left.\mathrm{ArCH}_{2} \mathrm{CH}_{2} \mathrm{CH}_{2}, \mathrm{OCH}_{2} \mathrm{CH}\left(\mathrm{CH}_{2} \mathrm{OC}=\mathrm{ONHR}\right) \mathrm{O}\right)$, 3.82-4.19 (m, $\left.\mathrm{OCH}_{2} \mathrm{CH}\left(\mathrm{CH}_{2} \mathrm{OC}=\mathrm{ONHR}\right) \mathrm{O}\right), 7.10-7.30\left(\mathrm{~m}, \mathrm{ArCH}_{2} \mathrm{CH}_{2}\right)$ ppm. ${ }^{13} \mathrm{C} \mathrm{NMR}(101$ MHz, DMSO-d 6 ) (5): $\delta=13.8\left(\mathrm{NHCH}_{2} \mathrm{CH}_{2}\left(\mathrm{CH}_{2}\right)_{9} \mathrm{CH}_{3}\right), 22.0-28.9\left(\mathrm{NHCH}_{2}\left(\mathrm{CH}_{2}\right)_{10} \mathrm{CH}_{3}\right), 26.2$ $\left(\mathrm{NHCH}_{2} \mathrm{CH}_{2} \mathrm{CH}_{2} \mathrm{~N}^{+}\left(\mathrm{CH}_{3}\right)_{3}\right), \quad 31.2\left(\mathrm{NHCH}_{2}\left(\mathrm{CH}_{2}\right)_{10} \mathrm{CH}_{3}\right), \quad 37.3 \quad\left(\mathrm{NHCH}_{2} \mathrm{CH}_{2} \mathrm{CH}_{2} \mathrm{~N}^{+}\left(\mathrm{CH}_{3}\right)_{3}\right)$, $52.2\left(\mathrm{NHCH}_{2} \mathrm{CH}_{2} \mathrm{CH}_{2} \mathrm{~N}^{+}\left(\mathrm{CH}_{3}\right)_{3}\right)$, $63.2\left(\mathrm{NHCH}_{2} \mathrm{CH}_{2} \mathrm{CH}_{2} \mathrm{~N}^{+}\left(\mathrm{CH}_{3}\right)_{3}\right)$, 68.6-77.0 $\left(\mathrm{ArCH}_{2} \mathrm{CH}_{2} \mathrm{CH}_{2}\right.$, $\left.\left.\mathrm{OCH}_{2} \mathrm{CH}\left(\mathrm{CH}_{2} \mathrm{OC}=\mathrm{ONHR}\right) \mathrm{O}\right), \mathrm{OCH}_{2} \mathrm{CH}\left(\mathrm{CH}_{2} \mathrm{OC}=\mathrm{ONHR}\right) \mathrm{O}\right), 125.5\left(\mathrm{ArCH}_{2}\right), 128.9\left(\mathrm{ArCH}_{2}\right), 141.5$ $\left(\mathrm{ArCH} \mathrm{CH}_{2}\right), 156.0\left(\mathrm{OCH}_{2} \mathrm{CH}\left(\mathrm{CH}_{2} \mathrm{OC}=\mathrm{ONHR}\right) \mathrm{O}\right) \mathrm{ppm}$.

2.10. Synthesis of Poly(glycidyl 3-aminopropyldiethanolmethylcarbamate-co-glycidyl dodecylcarbamate) $\left(P\left(G^{A P D E M A}{ }_{16}-\mathrm{CO}-G^{D D A}{ }_{11}\right)\right)(6)$

$\mathrm{P}\left(\mathrm{G}^{\mathrm{APDEA}}{ }_{16}\right.$-co- $\left.^{\mathrm{DDA}}{ }_{11}\right)(4)\left(0.216 \mathrm{~g}, 0.47 \mathrm{mmol}-\mathrm{NEtOH}_{2}\right)$ was dissolved in THF $(2.5 \mathrm{~mL})$. Methyl iodide $(0.081 \mathrm{~g}, 0.57 \mathrm{mmol})$ was added and the solution was stirred for $20 \mathrm{~h}$ at room temperature. Excess methyl iodide and the solvent were removed under reduced pressure and the polymer purified by dialysis in methanol. Polymer 6 was obtained as a slightly yellow solid $(0.238 \mathrm{~g}, 84 \%) . M_{n, \mathrm{NMR}}=9608 \mathrm{~g} \cdot \mathrm{mol}^{-1}, M_{\mathrm{n}, \mathrm{SEC}}=$ not measurable. ${ }^{1} \mathrm{H}$ NMR (400 MHz, MeOD) (6): $\delta=0.96\left(\mathrm{t},{ }^{3} J_{\mathrm{HH}}=6.3 \mathrm{~Hz}, \mathrm{NHCH}_{2} \mathrm{CH}_{2}\left(\mathrm{CH}_{2}\right)_{9} \mathrm{CH}_{3}\right)$, 1.35 (s, $\left.\mathrm{NHCH}_{2} \mathrm{CH}_{2}\left(\mathrm{CH}_{2}\right)_{9} \mathrm{CH}_{3}\right), \quad 1.48-1.65$ (m, $\left.\mathrm{NHCH}_{2} \mathrm{CH}_{2}\left(\mathrm{CH}_{2}\right)_{9} \mathrm{CH}_{3}\right), \quad 1.88-1.99$ (m, $\left.\mathrm{ArCH}_{2} \mathrm{CH}_{2}\right), 2.05-2.23\left(\mathrm{~m}, \mathrm{NHCH}_{2} \mathrm{CH}_{2} \mathrm{CH}_{2} \mathrm{~N}^{+}\left(\mathrm{CH}_{3}\left(\mathrm{CH}_{2} \mathrm{CH}_{2} \mathrm{OH}\right)_{2}\right)\right), 2.70-2.78\left(\mathrm{~m}, \mathrm{ArCH}_{2} \mathrm{CH}_{2}\right)$, 3.09-3.20 (m, $\left.\mathrm{NHCH}_{2} \mathrm{CH}_{2}\left(\mathrm{CH}_{2}\right)_{9} \mathrm{CH}_{3}\right)$, 3.24-3.42 (m, $\mathrm{NHCH}_{2} \mathrm{CH}_{2} \mathrm{CH}_{2} \mathrm{~N}^{+}\left(\mathrm{CH}_{3}\left(\mathrm{CH}_{2} \mathrm{CH}_{2} \mathrm{OH}\right)_{2}\right)$ ), 3.51-3.96 (m, $\left.\mathrm{ArCH}_{2} \mathrm{CH}_{2} \mathrm{CH}_{2}, \quad \mathrm{OCH}_{2} \mathrm{CH}\left(\mathrm{CH}_{2} \mathrm{OC}=\mathrm{ONHR}\right) \mathrm{O}, \quad \mathrm{CH}_{2} \mathrm{~N}^{+}\left(\mathrm{CH}_{3}\left(\mathrm{CH}_{2} \mathrm{CH}_{2} \mathrm{OH}\right)_{2}\right)\right)$, 4.02-4.42 (m, $\left.\quad \mathrm{OCH}_{2} \mathrm{CH}\left(\mathrm{CH}_{2} \mathrm{OC}=\mathrm{ONHR}\right) \mathrm{O}, \quad \mathrm{CH}_{2} \mathrm{~N}^{+}\left(\mathrm{CH}_{3}\left(\mathrm{CH}_{2} \mathrm{CH}_{2} \mathrm{OH}\right)_{2}\right)\right), \quad 7.20-7.36 \quad(\mathrm{~m}$, $\left.A r \mathrm{CH}_{2} \mathrm{CH}_{2}\right)$ ppm. ${ }^{13} \mathrm{C} \mathrm{NMR}(101 \mathrm{MHz}, \mathrm{MeOD})(6): \delta=14.5\left(\mathrm{NHCH}_{2} \mathrm{CH}_{2}\left(\mathrm{CH}_{2}\right)_{9} \mathrm{CH}_{3}\right)$, 23.7-31.0 ( $\left.\mathrm{NHCH}_{2} \mathrm{CH}_{2}\left(\mathrm{CH}_{2}\right)_{9} \mathrm{CH}_{3}\right), \quad 28.0 \quad\left(\mathrm{NHCH}_{2} \mathrm{CH}_{2} \mathrm{CH}_{2} \mathrm{~N}^{+}\left(\mathrm{CH}_{3}\left(\mathrm{CH}_{2} \mathrm{CH}_{2} \mathrm{OH}\right)_{2}\right), \quad 33.0\right.$ $\left(\mathrm{NHCH}_{2} \mathrm{CH}_{2}\left(\mathrm{CH}_{2}\right)_{9} \mathrm{CH}_{3}\right), 38.9\left(\mathrm{NHCH}_{2} \mathrm{CH}_{2} \mathrm{CH}_{2} \mathrm{~N}^{+}\left(\mathrm{CH}_{3}\left(\mathrm{CH}_{2} \mathrm{CH}_{2} \mathrm{OH}\right)_{2}\right)\right), 42.0\left(\mathrm{NHCH}_{2} \mathrm{CH}_{2}\left(\mathrm{CH}_{2}\right)_{9} \mathrm{CH}_{3}\right)$, $50.9\left(\mathrm{NHCH}_{2} \mathrm{CH}_{2} \mathrm{CH}_{2} \mathrm{~N}^{+}\left(\mathrm{CH}_{3}\left(\mathrm{CH}_{2} \mathrm{CH}_{2} \mathrm{OH}\right)_{2}\right), 56.8\left(\mathrm{NHCH}_{2} \mathrm{CH}_{2} \mathrm{CH}_{2} \mathrm{~N}^{+}\left(\mathrm{CH}_{3}\left(\mathrm{CH}_{2} \mathrm{CH}_{2} \mathrm{OH}\right)_{2}\right), 62.8-79.1\right.\right.$ $\left(\mathrm{ArCH}_{2} \mathrm{CH}_{2} \mathrm{CH}_{2}, \mathrm{OCH}_{2} \mathrm{CH}\left(\mathrm{CH}_{2} \mathrm{OC}=\mathrm{ONHR}\right) \mathrm{O}\right), 65.4\left(\mathrm{CH}_{2} \mathrm{~N}^{+}\left(\mathrm{CH}_{3}\left(\mathrm{CH}_{2} \mathrm{CH}_{2} \mathrm{OH}\right)_{2}\right), 129.4\left(\mathrm{ArCH}_{2}\right), 129.6\right.$ $\left(\mathrm{ArCH}_{2}\right), 158.7\left(\mathrm{OCH}_{2} \mathrm{CH}\left(\mathrm{CH}_{2} \mathrm{OC}=\mathrm{ONHR}\right) \mathrm{O}\right) \mathrm{ppm}$.

\subsection{Synthesis of Poly(glycidyl-4-nitrophenyl carbonate) $\left(P\left(G^{N P C}\right)_{27}\right)(7)$}

$\mathrm{PG}_{27}$ (1) (2.012 g, $\left.27.16 \mathrm{mmol} \mathrm{OH}\right)$ was dissolved in pyridine (18.85 mL). The 4-nitrophenyl chloroformate $(6.023 \mathrm{~g}, 29.88 \mathrm{mmol})$ was dissolved in dichloromethane $(20 \mathrm{~mL})$ and added to the polymer solution via syringe pump in $30 \mathrm{~min}$ at $0{ }^{\circ} \mathrm{C}$. The solution was stirred for $20 \mathrm{~h}$ at room temperature. The crude product was washed with water $(30 \mathrm{~mL}), 1 \mathrm{M} \mathrm{HCl}$ (aq.) $(3 \times 30 \mathrm{~mL})$, and saturated $\mathrm{NaCl}$ solution (aq.) $\left(30 \mathrm{~mL}\right.$ ). The organic phase was separated, dried over $\mathrm{Na}_{2} \mathrm{SO}_{4}$ and precipitated in cold $\mathrm{MeOH}$. The solvent was removed under reduced pressure and polymer 7 was obtained as a colorless solid $(5.782 \mathrm{~g}, 89 \%) \cdot M_{n, N M R}=6458 \mathrm{~g} \cdot \mathrm{mol}^{-1}, M_{n, S E C}=6600 \mathrm{~g} \cdot \mathrm{mol}^{-1}$, 
$Ð=$ 1.41. ${ }^{1} \mathrm{H}$ NMR $\left(400 \mathrm{MHz}, \mathrm{DMSO}-d_{6}\right)(7): \delta=1.69-1.83\left(\mathrm{~m}, \mathrm{ArCH}_{2} \mathrm{CH}_{2}\right), 2.54-2.61\left(\mathrm{~m}, \mathrm{ArCH}_{2} \mathrm{CH}_{2}\right)$, 3.54-3.95 (m, $\left.\mathrm{ArCH}_{2} \mathrm{CH}_{2} \mathrm{CH}_{2}, \mathrm{OCH}_{2} \mathrm{CH}\left(\mathrm{CH}_{2} \mathrm{OC}=\mathrm{OOArNO}_{2}\right) \mathrm{O}\right)$, 4.15-4.56 (d, $\left.\mathrm{CH}_{2} \mathrm{OC}=\mathrm{OOArNO}_{2}\right)$, 7.08-7.25 (m, $\left.A r \mathrm{CH}_{2} \mathrm{CH}_{2}\right), 7.42\left(\mathrm{~s}, \mathrm{CH}_{2} \mathrm{OC}=\mathrm{OO} A r \mathrm{NO}_{2}\right), 8.18\left(\mathrm{~s}, \mathrm{CH}_{2} \mathrm{OC}=\mathrm{OO} \operatorname{ArNO} \mathrm{NO}_{2}\right)$ ppm. ${ }^{13} \mathrm{C} \mathrm{NMR}$ $\left(101 \mathrm{MHz}, \mathrm{DMSO}-d_{6}\right)(7): \delta=30.8\left(\mathrm{ArCH}_{2} \mathrm{CH}_{2}\right), 31.6\left(\mathrm{ArCH}_{2}\right), 68.2\left(\mathrm{OCH}_{2} \mathrm{CH}\left(\mathrm{CH}_{2} \mathrm{OC}=\mathrm{OOArNO}_{2}\right) \mathrm{O}\right)$, $76.4\left(\mathrm{OCH}_{2} \mathrm{CH}\left(\mathrm{CH}_{2} \mathrm{OC}=\mathrm{OOArNO}{ }_{2}\right) \mathrm{O}\right), 76.5\left(\mathrm{ArCH}_{2} \mathrm{CH}_{2} \mathrm{CH}_{2}\right), 76.6\left(\mathrm{OCH}_{2} \mathrm{CH}\left(\mathrm{CH}_{2} \mathrm{OC}=\mathrm{OOArNO}{ }_{2}\right) \mathrm{O}\right)$, 122.3 $\left.\left.\left(\mathrm{CH}_{2} \mathrm{OC}=\mathrm{OO} A r \mathrm{NO}_{2}\right), 125.2\left(\mathrm{CH}_{2} \mathrm{OC}=\mathrm{OO} A r \mathrm{NO}_{2}\right), 125.7\left(\operatorname{ArCH} \mathrm{CH}_{2}\right), 128.2(\operatorname{ArCH})_{2}\right), 128.3(\operatorname{ArCH})_{2}\right)$ 141.6 $\left(A r \mathrm{CH}_{2}\right), 145.0\left(\mathrm{CH}_{2} \mathrm{OC}=\mathrm{OO} A r \mathrm{NO}_{2}\right), 152.0(\mathrm{OC}=\mathrm{OO}), 155.1\left(\mathrm{CH}_{2} \mathrm{OC}=\mathrm{OO} A r \mathrm{NO}_{2}\right)$ ppm.

\subsection{Synthesis of Poly(glycidyl homocysteine thiolactonylcarbamate) $\left(P\left(G^{H C T L}\right)_{27}\right)(8)$}

$\mathrm{P}\left(\mathrm{G}^{\mathrm{NPC}}\right)_{27}$ (7) $(1.020 \mathrm{~g}, 4.26 \mathrm{mmol}$ carbonate) was dissolved in DMF $(10 \mathrm{~mL})$, and 4-(dimethylamino)pyridine $(0.053 \mathrm{~g}, 0.43 \mathrm{mmol})$ and DL-homocysteine thiolactone hydrochloride $(0.655 \mathrm{~g}, 4.26 \mathrm{mmol})$ were added. The mixture was cooled to $0{ }^{\circ} \mathrm{C}$ and triethylamine $(0.862 \mathrm{~g}$, $8.52 \mathrm{mmol}$ ) was added over $1 \mathrm{~h}$ via syringe pump. The solution was stirred for $20 \mathrm{~h}$ at room temperature. DMF was removed under reduced pressure at $50{ }^{\circ} \mathrm{C}$ and the crude product was precipitated in $\mathrm{MeOH}$. Drying under reduced pressure at $50{ }^{\circ} \mathrm{C}$ gave polymer 8 as a slightly yellow solid $(0.694 \mathrm{~g}, 75 \%) . M_{\mathrm{n}, \mathrm{NMR}}=5865 \mathrm{~g} \cdot \mathrm{mol}^{-1}, M_{\mathrm{n}, \mathrm{SEC}}=6900 \mathrm{~g} \cdot \mathrm{mol}^{-1}, Ð=1.44 .{ }^{1} \mathrm{H} \mathrm{NMR}$ (400 MHz, DMSO- $\left.d_{6}\right)(8): \delta=1.74-1.83\left(\mathrm{~m}, \mathrm{ArCH}_{2} \mathrm{CH}_{2}\right), 2.00-2.48\left(\mathrm{~m}, \mathrm{C}=\mathrm{OSCH}_{2} \mathrm{CH}_{2} \mathrm{CHR}\right), 2.60$ $\left(\mathrm{t},{ }^{3} J_{\mathrm{H}, \mathrm{H}}=7.5 \mathrm{~Hz}, \mathrm{ArCH}_{2} \mathrm{CH}_{2}\right), 3.17-3.45\left(\mathrm{~m}, \mathrm{C}=\mathrm{OSCH}_{2} \mathrm{CH}_{2} \mathrm{CHR}\right), 3.47-3.75\left(\mathrm{~m}, \mathrm{ArCH}_{2} \mathrm{CH}_{2} \mathrm{CH}_{2}\right.$, $\left.\mathrm{OCH}_{2} \mathrm{CH}\left(\mathrm{CH}_{2} \mathrm{OC}=\mathrm{ONHR}\right) \mathrm{O}\right), 3.86-4.23\left(\mathrm{~m}, \mathrm{CH}_{2} \mathrm{OC}=\mathrm{ONHR}\right), 4.26-4.42\left(\mathrm{~m}, \mathrm{C}=\mathrm{OSCH}_{2} \mathrm{CH}_{2} \mathrm{CHR}\right)$, 7.14-7.29 (Ar), 7.55 (br s, NH) ppm. $\left.{ }^{13} \mathrm{C} \mathrm{NMR} \mathrm{(101} \mathrm{MHz,} \mathrm{DMSO-} d_{6}\right)(8): \delta=26.5\left(\mathrm{C}=\mathrm{OSCH}_{2} \mathrm{CH}_{2} \mathrm{CHR}\right)$, $29.8\left(\mathrm{C}=\mathrm{OSCH}_{2} \mathrm{CH}_{2} \mathrm{CHR}\right), 30.9\left(\mathrm{ArCH}_{2} \mathrm{CH}_{2}\right), 31.6\left(\mathrm{ArCH}_{2}\right), 59.9\left(\mathrm{CH}_{2} \mathrm{OC}=\mathrm{ONHR}\right), 63.7,68.7,77.2$ $\left(\mathrm{ArCH}_{2} \mathrm{CH}_{2} \mathrm{CH}_{2}, \mathrm{OCH}_{2} \mathrm{CH}\left(\mathrm{CH}_{2} \mathrm{OC}=\mathrm{ONHR}\right) \mathrm{O}\right), 79.2\left(\mathrm{C}=\mathrm{OSCH}_{2} \mathrm{CH}_{2} \mathrm{CHR}\right), 128.3\left(\mathrm{ArCH}_{2}\right), 128.4$ $\left(\mathrm{ArCH}_{2}\right), 156.0(\mathrm{OC}=\mathrm{ONHR}), 205.7\left(\mathrm{C}=\mathrm{OSCH}_{2} \mathrm{CH}_{2} \mathrm{CHR}\right) \mathrm{ppm}$.

\subsection{Synthesis of $P\left(G^{D D A c}\right)_{27}(\mathbf{9})$}

To a mixture of $\mathrm{P}\left(\mathrm{G}^{\mathrm{HCTL}}\right)_{27}$ (8) $(0.301 \mathrm{~g}, 1.386 \mathrm{mmol}$ HCTL$)$ and dodecyl acrylate $(0.833 \mathrm{~g}$, $3.464 \mathrm{mmol})$ in chloroform $(3.0 \mathrm{~mL})$, 3-(dimethylamino)-1-propylamine $(0.353 \mathrm{~g}, 3.464 \mathrm{mmol})$ was added in $30 \mathrm{~min}$ via syringe pump at room temperature. The mixture was stirred for $20 \mathrm{~h}$ at room temperature. Chloroform was removed under reduced pressure. Dialysis in acetone gave 9 as a colorless, viscous liquid $(0.628 \mathrm{~g}, 81 \%) . M_{\mathrm{n}, \mathrm{NMR}}=15,115 \mathrm{~g} \cdot \mathrm{mol}^{-1}, M_{\mathrm{n}, \mathrm{SEC}}=15,300 \mathrm{~g} \cdot \mathrm{mol}^{-1}$, $\Xi=$ 1.36. ${ }^{1} \mathrm{H}$ NMR $\left(400 \mathrm{MHz}, \mathrm{CDCl}_{3}\right)(9): \delta=0.82\left(\mathrm{t},{ }^{3} \mathrm{~J}_{\mathrm{H}, \mathrm{H}}=6.8 \mathrm{~Hz}, \mathrm{CH}_{2} \mathrm{CH}_{2}\left(\mathrm{CH}_{2}\right)_{9} \mathrm{CH}_{3}\right)$, $1.20\left(\mathrm{~s}, \mathrm{CH}_{2} \mathrm{CH}_{2}\left(\mathrm{CH}_{2}\right)_{9} \mathrm{CH}_{3}\right), 1.48-1.69\left(\mathrm{~m}, \mathrm{CH}_{2} \mathrm{CH}_{2} \mathrm{~N}\left(\mathrm{CH}_{3}\right)_{2}, \mathrm{CH}_{2} \mathrm{CH}_{2}\left(\mathrm{CH}_{2}\right)_{9} \mathrm{CH}_{3}, \mathrm{ArCH}_{2} \mathrm{CH}_{2}\right)$, 1.76-2.08 (m, $\left.\mathrm{CHCH}_{2} \mathrm{CH}_{2} \mathrm{SCH}_{2}\right), 2.15\left(\mathrm{~s}, \mathrm{CH}_{2} \mathrm{~N}\left(\mathrm{CH}_{3}\right)_{2}\right), 2.22-2.37\left(\mathrm{~m}, \mathrm{CH}_{2} \mathrm{~N}\left(\mathrm{CH}_{3}\right)_{2}\right), 2.43-2.61(\mathrm{~m}$, $\left.\mathrm{CHCH}_{2} \mathrm{CH}_{2} \mathrm{SCH}_{2} \mathrm{CH}_{2}\right), 2.64-2.78\left(\mathrm{~m}, \mathrm{CHCH}_{2} \mathrm{CH}_{2} \mathrm{SCH}_{2} \mathrm{CH}_{2}\right), 3.07-3.37$ (m, NHCH $\left.\left.\mathrm{CH}_{2} \mathrm{CH}_{2} \mathrm{~N}_{(} \mathrm{CH}_{3}\right)_{2}\right)$, 3.41-3.75 (m, $\left.\mathrm{ArCH}_{2} \mathrm{CH}_{2} \mathrm{CH}_{2}, \mathrm{OCH}_{2} \mathrm{CH}\left(\mathrm{CH}_{2} \mathrm{OC}=\mathrm{ONHR}\right) \mathrm{O}\right)$, 3.77-4.07 (m, $\left.\mathrm{CH}_{2} \mathrm{OC}=\mathrm{ONHCH}\right)$, 4.10-4.41 (m, O=COCH $\left.\mathrm{CH}_{2}\right), 7.09-7.23$ (m, Ar), 7.76 (br. s, NH) ppm. ${ }^{13} \mathrm{C} \mathrm{NMR}(101 \mathrm{MHz}$, $\left.\mathrm{CDCl}_{3}\right)(9): \delta=14.1\left(\mathrm{CH}_{2} \mathrm{CH}_{2}\left(\mathrm{CH}_{2}\right)_{9} \mathrm{CH}_{3}\right), 22.7-31.9\left(\mathrm{CH}_{2} \mathrm{CH}_{2}\left(\mathrm{CH}_{2}\right)_{9} \mathrm{CH}_{3}, \mathrm{CHCH}_{2} \mathrm{CH}_{2} \mathrm{SCH}_{2}\right.$, $\left.\mathrm{ArCH}_{2} \mathrm{CH}_{2}\right), 26.8\left(\mathrm{CHCH}_{2} \mathrm{CH}_{2} \mathrm{SCH}_{2} \mathrm{CH}_{2}\right), 28.2\left(\mathrm{CH}_{2} \mathrm{CH}_{2} \mathrm{~N}\left(\mathrm{CH}_{3}\right)_{2}\right), 28.6\left(\mathrm{CHCH}_{2} \mathrm{CH}_{2} \mathrm{SCH}_{2} \mathrm{CH}_{2}\right), 34.7$ $\left(\mathrm{CHCH}_{2} \mathrm{CH}_{2} \mathrm{SCH}_{2} \mathrm{CH}_{2}\right), 38.7\left(\mathrm{NHCH}_{2} \mathrm{CH}_{2} \mathrm{CH}_{2} \mathrm{~N}\left(\mathrm{CH}_{3}\right)_{2}\right), 45.5\left(\mathrm{CH}_{2} \mathrm{~N}\left(\mathrm{CH}_{3}\right)_{2}\right), 54.1(\mathrm{NHCH}), 57.9$ $\left.\left(\mathrm{CH}_{2} \mathrm{~N}\left(\mathrm{CH}_{3}\right)_{2}\right), 64.9\left(\mathrm{O}=\mathrm{COCH}_{2} \mathrm{CH}_{2}\right), 125.9\left(\mathrm{ArCH}_{2}\right), 128.3\left(\mathrm{ArCH} \mathrm{CH}_{2}\right), 128.5\left(\mathrm{ArCH} \mathrm{CH}_{2}\right), 140.0(\mathrm{ArCH})_{2}\right)$, $156.4(\mathrm{OC}=\mathrm{ONH}), 171.5(\mathrm{CHC}=\mathrm{ONH}), 172.0\left(\mathrm{CH}_{2} \mathrm{C}=\mathrm{OO}\right) \mathrm{ppm}$.

\subsection{Synthesis of $P\left(G^{D D A c, q}\right)_{27}(\mathbf{1 0})$}

$\mathrm{P}\left(\mathrm{G}^{\mathrm{DDAc}}\right)_{27}$ (9) $\left(0.431 \mathrm{~g}, 0.770 \mathrm{mmol}-\mathrm{NMe}_{2}\right)$ was dissolved in THF $(9.0 \mathrm{~mL})$, and methyl iodide $(0.131 \mathrm{~g}, 0.923 \mathrm{mmol})$ was added. The solution was stirred at room temperature for $20 \mathrm{~h}$. Removal of THF and excess methyl iodide under reduced pressure and dialysis in methanol gave polymer $\mathbf{1 0}$ as a slightly yellow solid $(0.475 \mathrm{~g}, 88 \%) \cdot M_{\mathrm{n}, \mathrm{NMR}}=18,947 \mathrm{~g} \cdot \mathrm{mol}^{-1}, M_{\mathrm{n}, \mathrm{SEC}}=11,800 \mathrm{~g} \cdot \mathrm{mol}^{-1}, Ð=1.36$. ${ }^{1} \mathrm{H}$ NMR $\left(400 \mathrm{MHz}, \mathrm{CDCl}_{3}\right)(\mathbf{1 0}): \delta=0.81\left(\mathrm{t},{ }^{3} J_{\mathrm{H}, \mathrm{H}}=6.5 \mathrm{~Hz}, \mathrm{CH}_{2} \mathrm{CH}_{2}\left(\mathrm{CH}_{2}\right)_{9} \mathrm{CH}_{3}\right), 1.05-1.32(\mathrm{~m}$, $\left.\mathrm{CH}_{2} \mathrm{CH}_{2}\left(\mathrm{CH}_{2}\right)_{9} \mathrm{CH}_{3}\right), 1.46-1.62\left(\mathrm{~m}, \mathrm{CH}_{2} \mathrm{CH}_{2}\left(\mathrm{CH}_{2}\right)_{9} \mathrm{CH}_{3}\right), 1.85-2.25\left(\mathrm{~m}, \mathrm{CH}_{2} \mathrm{CH}_{2} \mathrm{~N}^{+}\left(\mathrm{CH}_{3}\right)_{3}, \mathrm{ArCH}_{2} \mathrm{CH}_{2}\right.$, 
$\left.\mathrm{CHCH}_{2} \mathrm{CH}_{2} \mathrm{SCH}_{2}\right), 2.39-2.64\left(\mathrm{~m}, \mathrm{CHCH}_{2} \mathrm{CH}_{2} \mathrm{SCH}_{2} \mathrm{CH}_{2}\right), 2.66-2.82\left(\mathrm{~m}, \mathrm{CHCH}_{2} \mathrm{CH}_{2} \mathrm{SCH}_{2} \mathrm{CH}_{2}\right)$, 3.05-3.50 (m, $\left.\mathrm{NHCH}_{2} \mathrm{CH}_{2} \mathrm{CH}_{2} \mathrm{~N}^{+}\left(\mathrm{CH}_{3}\right)_{3}\right), 3.51-3.83\left(\mathrm{~m}, \mathrm{ArCH}_{2} \mathrm{CH}_{2} \mathrm{CH}_{2}, \mathrm{OCH}_{2} \mathrm{CH}\left(\mathrm{CH}_{2} \mathrm{OC}=\mathrm{ONHR}\right) \mathrm{O}\right)$, 3.84-4.50 (m, $\left.\mathrm{CH}_{2} \mathrm{OC}=\mathrm{ONHCH}, \mathrm{O}=\mathrm{COCH}_{2} \mathrm{CH}_{2}\right), 7.08-7.24$ (m, Ar), 7.77 (br. s, NH) ppm. ${ }^{13} \mathrm{C} \mathrm{NMR}(101$ $\left.\mathrm{MHz}, \mathrm{CDCl}_{3}\right)(\mathbf{1 0}): \delta=14.1\left(\mathrm{CH}_{2} \mathrm{CH}_{2}\left(\mathrm{CH}_{2}\right)_{9} \mathrm{CH}_{3}\right), 22.6-31.9\left(\mathrm{CH}_{2} \mathrm{CH}_{2}\left(\mathrm{CH}_{2}\right)_{9} \mathrm{CH}_{3}, \mathrm{CHCH}_{2} \mathrm{CH}_{2} \mathrm{SCH}_{2}\right.$, $\left.\mathrm{ArCH}_{2} \mathrm{CH}_{2}\right), 26.7\left(\mathrm{CHCH}_{2} \mathrm{CH}_{2} \mathrm{SCH}_{2} \mathrm{CH}_{2}\right), 28.3\left(\mathrm{CH}_{2} \mathrm{CH}_{2} \mathrm{~N}^{+}\left(\mathrm{CH}_{3}\right)_{3}\right), 28.6\left(\mathrm{CHCH}_{2} \mathrm{CH}_{2} \mathrm{SCH}_{2} \mathrm{CH}_{2}\right)$, $34.8\left(\mathrm{CHCH}_{2} \mathrm{CH}_{2} \mathrm{SCH}_{2} \mathrm{CH}_{2}\right), 40.1\left(\mathrm{NHCH}_{2} \mathrm{CH}_{2} \mathrm{CH}_{2} \mathrm{~N}^{+}\left(\mathrm{CH}_{3}\right)_{3}\right), 53.8\left(\mathrm{CH}_{2} \mathrm{~N}^{+}\left(\mathrm{CH}_{3}\right)_{3}, \mathrm{NHCH}\right), 64.9$ $\left(\mathrm{CH}_{2} \mathrm{~N}^{+}\left(\mathrm{CH}_{3}\right)_{3}, \mathrm{O}=\mathrm{COCH}_{2} \mathrm{CH}_{2}\right), 156.5(\mathrm{OC}=\mathrm{ONH}), 172.1(\mathrm{CHC}=\mathrm{ONH}), 172.8\left(\mathrm{CH}_{2} \mathrm{C}=\mathrm{OO}\right) \mathrm{ppm}$.

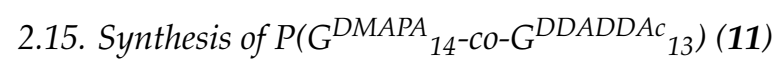

$\mathrm{P}\left(\mathrm{G}^{\mathrm{NPC}}\right)_{27}$ (7) (0.541 $\mathrm{g}, 2.26 \mathrm{mmol}$ carbonate) was dissolved in DMF (11 mL) and 3-(dimethylamino)-1-propylamine $(0.115 \mathrm{~g}, 1.13 \mathrm{mmol})$ was added in $30 \mathrm{~min}$ via syringe pump. The mixture was stirred for $20 \mathrm{~h}$ at room temperature. DL-homocysteine thiolactone hydrochloride $(0.174 \mathrm{~g}, 1.13 \mathrm{mmol})$ and 4 -(dimethylamino)pyridine $(0.013 \mathrm{~g}, 0.11 \mathrm{mmol})$ were added. The mixture was cooled to $0{ }^{\circ} \mathrm{C}$, and triethylamine $(0.229 \mathrm{~g}, 2.26 \mathrm{mmol})$ was added over $1 \mathrm{~h}$ via syringe pump. The solution was stirred for $20 \mathrm{~h}$ at room temperature. DMF was removed under reduced pressure at room temperature. The crude product was dissolved in chloroform, and dodecyl acrylate $(0.680 \mathrm{~g}$, $2.83 \mathrm{mmol})$ was added. The mixture was cooled to $0{ }^{\circ} \mathrm{C}$, and dodecylamine $(0.525 \mathrm{~g}, 2.83 \mathrm{mmol})$ was added in $30 \mathrm{~min}$ using a syringe pump. After stirring for $20 \mathrm{~h}$ at room temperature, the solvent was removed and the crude product was purified by dialysis in acetone. Polymer $\mathbf{1 1}$ was received as a slightly yellow, viscous liquid $(0.469 \mathrm{~g}, 48 \%) \cdot M_{\mathrm{n}, \mathrm{NMR}}=11,631 \mathrm{~g} \cdot \mathrm{mol}^{-1}, M_{\mathrm{n}, \mathrm{SEC}}=10,800 \mathrm{~g} \cdot \mathrm{mol}^{-1}$, $Ð=1.36 . \quad{ }^{1} \mathrm{H}$ NMR $\left(400 \mathrm{MHz}, \mathrm{CDCl}_{3} /\right.$ acetone- $\left.d_{6}(6: 4)\right)(\mathbf{1 1}): \delta=0.81\left(\mathrm{t},{ }^{3} J_{H, H}=6.6 \mathrm{~Hz}\right.$ $\left.\mathrm{CH}_{2} \mathrm{CH}_{2}\left(\mathrm{CH}_{2}\right)_{9} \mathrm{CH}_{3}\right), 1.20$ (s, $\left.\mathrm{CH}_{2} \mathrm{CH}_{2}\left(\mathrm{CH}_{2}\right)_{9} \mathrm{CH}_{3}\right), 1.38-1.50$ (m, $\left.\mathrm{NHCH}_{2} \mathrm{CH}_{2}\left(\mathrm{CH}_{2}\right)_{9} \mathrm{CH}_{3}\right), 1.51-1.64$ $\left(\mathrm{m}, \mathrm{OCH}_{2} \mathrm{CH}_{2}\left(\mathrm{CH}_{2}\right)_{9} \mathrm{CH}_{3}\right), 1.70-1.86\left(\mathrm{~m}, \mathrm{CH}_{2} \mathrm{CH}_{2} \mathrm{~N}\left(\mathrm{CH}_{3}\right)_{2}\right), 1.88-2.01$ (m, $\left.\mathrm{CHCH}_{2} \mathrm{CH}_{2} \mathrm{SCH}_{2}\right)$, 2.29-2.77 (m, $\left.\mathrm{CH}_{2} \mathrm{~N}\left(\mathrm{CH}_{3}\right)_{2}, \mathrm{CHCH}_{2} \mathrm{CH}_{2} \mathrm{SCH}_{2} \mathrm{CH}_{2}\right), 2.97-3.30$ (m, $\mathrm{NHCH}_{2} \mathrm{CH}_{2} \mathrm{CH}_{2} \mathrm{~N}\left(\mathrm{CH}_{3}\right)_{2}$, $\left.\mathrm{NHCH}_{2} \mathrm{CH}_{2}\left(\mathrm{CH}_{2}\right)_{9} \mathrm{CH}_{3}\right), 3.44-3.79\left(\mathrm{~m}, \mathrm{ArCH}_{2} \mathrm{CH}_{2} \mathrm{CH}_{2}, \mathrm{OCH}_{2} \mathrm{CH}\left(\mathrm{CH}_{2} \mathrm{OC}=\mathrm{ONHR}\right) \mathrm{O}\right), 3.90-4.34$ $\left(\mathrm{m}, \quad \mathrm{CH}_{2} \mathrm{OC}=\mathrm{ONHR}, \quad \mathrm{CH}_{2} \mathrm{OC}=\mathrm{ONHCH}, \mathrm{O}=\mathrm{COCH}_{2} \mathrm{CH}_{2}\right), \quad 7.07-7.24(\mathrm{~m}, \mathrm{Ar})$ ppm. ${ }^{13} \mathrm{C}$ NMR (101 MHz, $\mathrm{CDCl}_{3} /$ acetone- $d_{6}$ (6:4)) (11): $\delta=13.5\left(\mathrm{CH}_{2} \mathrm{CH}_{2}\left(\mathrm{CH}_{2}\right)_{9} \mathrm{CH}_{3}\right), \quad 22.1-31.4$ $\left(\mathrm{CH}_{2} \mathrm{CH}_{2}\left(\mathrm{CH}_{2}\right)_{9} \mathrm{CH}_{3}, \mathrm{CHCH}_{2} \mathrm{CH}_{2} \mathrm{SCH}_{2}, \mathrm{ArCH}_{2} \mathrm{CH}_{2}, \mathrm{CH}_{2} \mathrm{CH}_{2} \mathrm{~N}\left(\mathrm{CH}_{3}\right)_{2}\right), 34.2\left(\mathrm{CHCH}_{2} \mathrm{CH}_{2} \mathrm{SCH}_{2} \mathrm{CH}_{2}\right)$, $38.4\left(\mathrm{NHCH}_{2} \mathrm{CH}_{2} \mathrm{CH}_{2} \mathrm{~N}\left(\mathrm{CH}_{3}\right)_{2}\right), 39.0\left(\mathrm{NHCH}_{2} \mathrm{CH}_{2}\left(\mathrm{CH}_{2}\right)_{9} \mathrm{CH}_{3}\right), 43.7\left(\mathrm{CH}_{2} \mathrm{~N}\left(\mathrm{CH}_{3}\right)_{2}\right), 53.3(\mathrm{NHCH})$, $56.0\left(\mathrm{CH}_{2} \mathrm{~N}\left(\mathrm{CH}_{3}\right)_{2}\right), 64.2\left(\mathrm{O}=\mathrm{COCH}_{2} \mathrm{CH}_{2}\right), 127.8\left(\mathrm{ArCH}_{2}\right), 127.9\left(\mathrm{ArCH}_{2}\right), 155.9(\mathrm{OC}=\mathrm{ONH}), 156.2$ $(\mathrm{OC}=\mathrm{ONH}), 171.4(\mathrm{CHC}=\mathrm{ONH}), 172.1\left(\mathrm{CH}_{2} \mathrm{C}=\mathrm{OO}\right) \mathrm{ppm}$.

2.16. Synthesis of $P\left(G^{T M A P A}{ }_{14}^{-c o-} G^{D D A D D A c} 13\right)(12)$

$\mathrm{P}\left(\mathrm{G}^{\mathrm{DMAPA}}{ }_{14-\mathrm{Co}-\mathrm{G}^{\mathrm{DDADDAc}}}{ }_{13}\right)(\mathbf{1 1})\left(0.179 \mathrm{~g}, 0.20 \mathrm{mmol}-\mathrm{NMe}_{2}\right)$ was dissolved in THF $(2.0 \mathrm{~mL})$, and methyl iodide $(0.036 \mathrm{~g}, 0.25 \mathrm{mmol})$ was added. The solution was stirred at room temperature for 20 h. Removal of THF and excess methyl iodide under reduced pressure and dialysis in methanol gave polymer 12 as a slightly yellow solid (0.203 g, quant.). $M_{\mathrm{n}, \mathrm{NMR}}=13,476 \mathrm{~g} \cdot \mathrm{mol}^{-1}, M_{\mathrm{n}, \mathrm{SEC}}=9400 \mathrm{~g} \cdot \mathrm{mol}^{-1}$, $Ð=1.41 .{ }^{1} \mathrm{H}-\mathrm{NMR}\left(400 \mathrm{MHz}, \mathrm{CDCl}_{3} /\right.$ acetone- $\left.d_{6}(6: 4)\right)(\mathbf{1 2}): \delta=0.80\left(\mathrm{t},{ }^{3} J_{\mathrm{H}, \mathrm{H}}=6.8 \mathrm{~Hz}\right.$ $\left.\mathrm{CH}_{2} \mathrm{CH}_{2}\left(\mathrm{CH}_{2}\right)_{9} \mathrm{CH}_{3}\right), 1.18\left(\mathrm{~s}, \mathrm{CH}_{2} \mathrm{CH}_{2}\left(\mathrm{CH}_{2}\right)_{9} \mathrm{CH}_{3}\right), 1.36-1.48\left(\mathrm{~m}, \mathrm{NHCH}_{2} \mathrm{CH}_{2}\left(\mathrm{CH}_{2}\right)_{9} \mathrm{CH}_{3}\right), 1.50-1.66$ (m, $\left.\mathrm{OCH}_{2} \mathrm{CH}_{2}\left(\mathrm{CH}_{2}\right)_{9} \mathrm{CH}_{3}\right), \quad$ 1.74-2.01 (m, $\left.\mathrm{CH}_{2} \mathrm{CH}_{2} \mathrm{~N}^{+}\left(\mathrm{CH}_{3}\right)_{3}, \mathrm{CHCH}_{2} \mathrm{CH}_{2} \mathrm{SCH}_{2}\right), \quad 2.42-2.89$ (m, $\left.\mathrm{CHCH}_{2} \mathrm{CH}_{2} \mathrm{SCH}_{2} \mathrm{CH}_{2}\right), 2.94-3.45\left(\mathrm{~m}, \mathrm{NHCH}_{2} \mathrm{CH}_{2} \mathrm{CH}_{2} \mathrm{~N}^{+}\left(\mathrm{CH}_{3}\right)_{3}, \mathrm{NHCH}_{2} \mathrm{CH}_{2}\left(\mathrm{CH}_{2}\right)_{9} \mathrm{CH}_{3}\right)$, 3.49-3.81 (m, $\left.\mathrm{ArCH}_{2} \mathrm{CH}_{2} \mathrm{CH}_{2}, \mathrm{OCH}_{2} \mathrm{CH}\left(\mathrm{CH}_{2} \mathrm{OC}=\mathrm{ONHR}\right) \mathrm{O}\right), 3.87-4.38$ (m, $\mathrm{CH}_{2} \mathrm{OC}=\mathrm{ONHR}$, $\left.\mathrm{CH}_{2} \mathrm{OC}=\mathrm{ONHCH}, \mathrm{O}=\mathrm{COCH}_{2} \mathrm{CH}_{2}\right), 7.03-7.23(\mathrm{~m}, \mathrm{Ar}) \mathrm{ppm} .{ }^{13} \mathrm{C} \mathrm{NMR}\left(101 \mathrm{MHz}, \mathrm{CDCl}_{3} /\right.$ acetone- $d_{6}$ (6:4)) (12): $\delta=13.4\left(\mathrm{CH}_{2} \mathrm{CH}_{2}\left(\mathrm{CH}_{2}\right)_{9} \mathrm{CH}_{3}\right), 22.1-31.3\left(\mathrm{CH}_{2} \mathrm{CH}_{2}\left(\mathrm{CH}_{2}\right)_{9} \mathrm{CH}_{3}, \mathrm{CHCH}_{2} \mathrm{CH}_{2} \mathrm{SCH}_{2}\right.$ ， $\left.\mathrm{ArCH}_{2} \mathrm{CH}_{2}, \mathrm{CH}_{2} \mathrm{CH}_{2} \mathrm{~N}\left(\mathrm{CH}_{3}\right)_{2}\right), 34.2\left(\mathrm{CHCH}_{2} \mathrm{CH}_{2} \mathrm{SCH}_{2} \mathrm{CH}_{2}\right), 38.9\left(\mathrm{NHCH}_{2} \mathrm{CH}_{2}\left(\mathrm{CH}_{2}\right)_{9} \mathrm{CH}_{3}\right), 43.0$ $\left(\mathrm{NHCH}_{2} \mathrm{CH}_{2} \mathrm{CH}_{2} \mathrm{~N}^{+}\left(\mathrm{CH}_{3}\right)_{2}\right), 53.0\left(\mathrm{CH}_{2} \mathrm{~N}^{+}\left(\mathrm{CH}_{3}\right)_{3}\right), 53.8(\mathrm{NHCH}), 64.1\left(\mathrm{CH}_{2} \mathrm{~N}\left(\mathrm{CH}_{3}\right)_{2}, 2 \mathrm{O}=\mathrm{COCH}_{2} \mathrm{CH}_{2}\right)$, 127.8 $\left(\mathrm{ArCH}_{2}\right), 127.9\left(\mathrm{ArCH} \mathrm{CH}_{2}\right), 141.3\left(\mathrm{ArCH}_{2}\right), 155.9(\mathrm{OC}=\mathrm{ONH}), 156.4(\mathrm{OC}=\mathrm{ONH}), 171.3(\mathrm{CHC}=\mathrm{ONH})$, $171.4\left(\mathrm{CH}_{2} \mathrm{C}=\mathrm{OO}\right) \mathrm{ppm}$. 


\section{Results and Discussion}

In the current work, we present the synthesis and characterization of various novel cationic-hydrophobic functionalized polyglycidols. The functional polyethers are evaluated in regard to their antibacterial activity against $E$. coli and $S$. aureus, induced by different microstructures.

Thereto, a polyglycidol with statistically distributed cationic and hydrophobic groups (cationic-hydrophobic ratio of 1:1) was compared to (a) a polyglycidol with a hydrophilic modification at the cationic moieties; (b) a polyglycidol with cationic and hydrophobic functionalities at every repeating unit; and (c) a polyglycidol with a cationic-hydrophobic balance of 1:2 (Figure 1).

Linear polyglycidol was synthesized by anionic ring-opening polymerization of ethoxyethyl glycidyl ether with 3-phenyl-1-propanol as initiator, followed by removal of the acetal protecting groups under acidic conditions [38]. Polyglycidol with 27 repeating units $\left(\mathrm{PG}_{27}(\mathbf{1})\right)$ and $M_{n, S E C}=2900 \mathrm{~g} \cdot \mathrm{mol}^{-1}$ was obtained with a narrow molecular weight distribution $(\nexists=1.13)$. The ${ }^{1} \mathrm{H},{ }^{13} \mathrm{C}$ NMR spectra and SEC analysis of $\mathrm{PG}_{27}(\mathbf{1})$ can be found in the supporting information (Figures S1-S3).

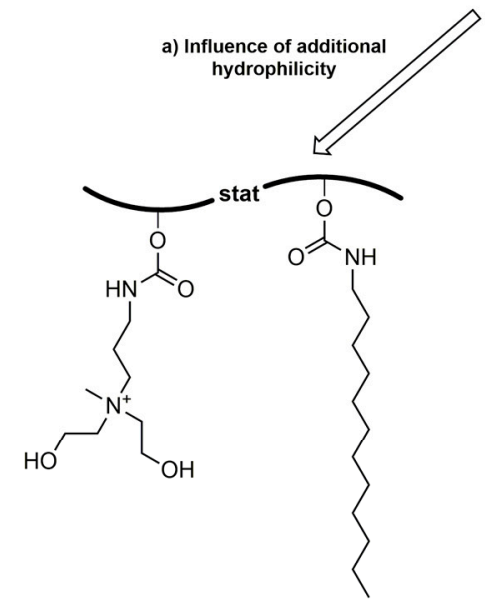

Cationic and hydrophobic groups
statistically distributed + hydrophilic statistically distributed + hydrophilic
modification at the cationic moiety
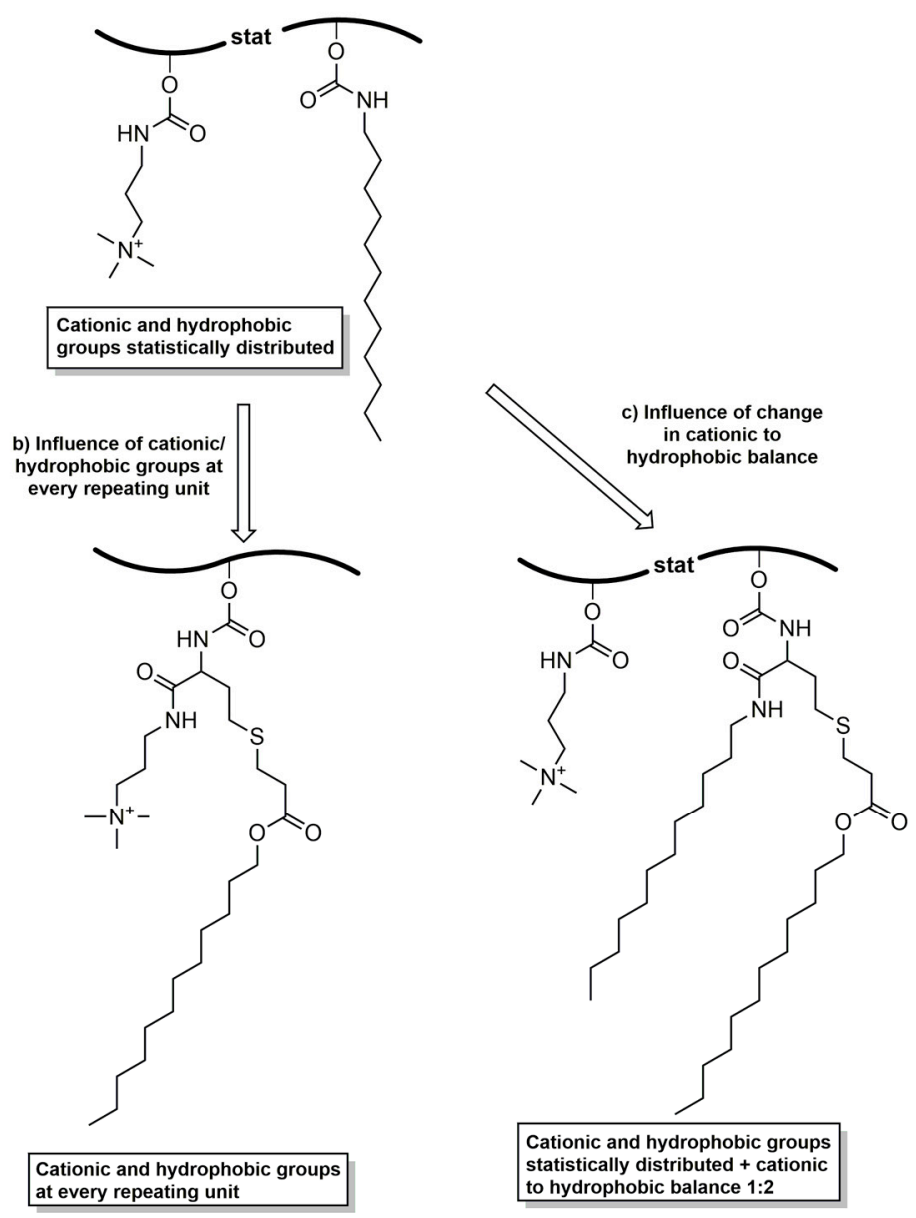

Figure 1. Comparison of various cationic-hydrophobic functionalized polyglycidols in regard to their antibacterial activity against $E$. coli and $S$. aureus to examine the structure-property relationship.

\subsection{Synthesis of $P\left(G^{T M A P A}{ }_{15-C o-}-G^{D D A}{ }_{12}\right)(5)$ and $P\left(G^{A P D E M A}{ }_{16}-C 0-G^{D D A}{ }_{11}\right)(6)$}

The general approach for the functionalization of linear polyglycidol with cationic and hydrophobic groups starts with the introduction of active ester functionalities to the polymer backbone. The active esters allow the reaction with primary amines under the selective formation of carbamate moieties. Quaternization of introduced tertiary amines gave the cationic component. 
$\mathrm{PG}_{27}$ (1) was reacted with phenyl chloroformate in pyridine/dichloromethane at room temperature (Scheme 1a). For purification, poly(glycidyl phenyl carbonate) ${ }_{27}$ (2) was washed with water, $1 \mathrm{M} \mathrm{HCl}$ solution (aq.), and saturated $\mathrm{NaCl}$ solution (aq.) to remove pyridine hydrochloride and excess pyridine. The successful functionalization was confirmed by ${ }^{1} \mathrm{H} N M R,{ }^{13} \mathrm{C} N M R$ spectroscopy, and SEC analysis (Figures S4-S6). The introduced phenyl carbonate groups are excellent electrophiles for the substitution reaction with non-functionalized, primary amines. $P\left(G^{\mathrm{PC}}\right)_{27}(2)$ was subsequently reacted with dodecylamine (DDA) and 3-(dimethylamino)-1-propylamine (DMAPA) or $N$-(3-aminopropyl)diethanolamine (APDEA) in THF at room temperature in a 1:1 ratio (Scheme 1b). The prepared poly(glycidyl 3-dimethylaminopropylcarbamate-co-glycidyl dodecylcarbamate) $\left(\mathrm{P}\left(\mathrm{G}^{\mathrm{DMAPA}}{ }_{15}-\mathrm{co}-\mathrm{G}^{\mathrm{DDA}}{ }_{12}\right)\right.$ ) (3) and poly(glycidyl 3-aminopropyldiethanolcarbamate-co-glycidyl dodecylcarbamate) ( $\left(\mathrm{G}^{\mathrm{APDEA}}{ }_{16}-\mathrm{co}-\mathrm{G}^{\mathrm{DDA}}{ }_{11}\right)$ ) (4) were purified by dialysis in methanol and characterized by ${ }^{1} \mathrm{H}$ NMR, ${ }^{13} \mathrm{C}$ NMR spectroscopy, and SEC analysis (Figures S7-S12). In both cases, the different functional groups are statistically distributed along the polyglycidol backbone. However, the higher reactivity of DMAPA and APDEA in comparison to DDA leads to a slightly uneven ratio of functionalities. $P\left(G^{D^{D A P A}}{ }_{15}-c o-G^{D D A}{ }_{12}\right)$ (3) and $\mathrm{P}\left(\mathrm{G}^{\mathrm{APDEA}}{ }_{16}-\mathrm{Co}-\mathrm{G}^{\mathrm{DDA}}{ }_{11}\right)$ (4) were further reacted with an excess of methyl iodide in THF at room temperature to quaternize the tertiary amine moieties (Scheme 1c). The synthesized poly(glycidyl 3-trimethylaminopropylcarbamate-co-glycidyl dodecylcarbamate) $\left(\mathrm{P}\left(\mathrm{G}^{\mathrm{TMAPA}}{ }_{15}-\mathrm{co}-\mathrm{G}^{\mathrm{DDA}} 12\right)\right)(5)$ and poly (glycidyl 3-aminopropyldiethanolmethylcarbamate-co-glycidyl dodecylcarbamate) (P(G $\left.\left.\mathrm{G}^{\mathrm{APDEMA}}{ }_{16}-\mathrm{co}-\mathrm{G}^{\mathrm{DDA}}{ }_{11}\right)\right)(\mathbf{6})$ were purified by dialysis in methanol and analyzed by ${ }^{1} \mathrm{H}$ NMR, ${ }^{13} \mathrm{C}$ NMR, and SEC analysis.

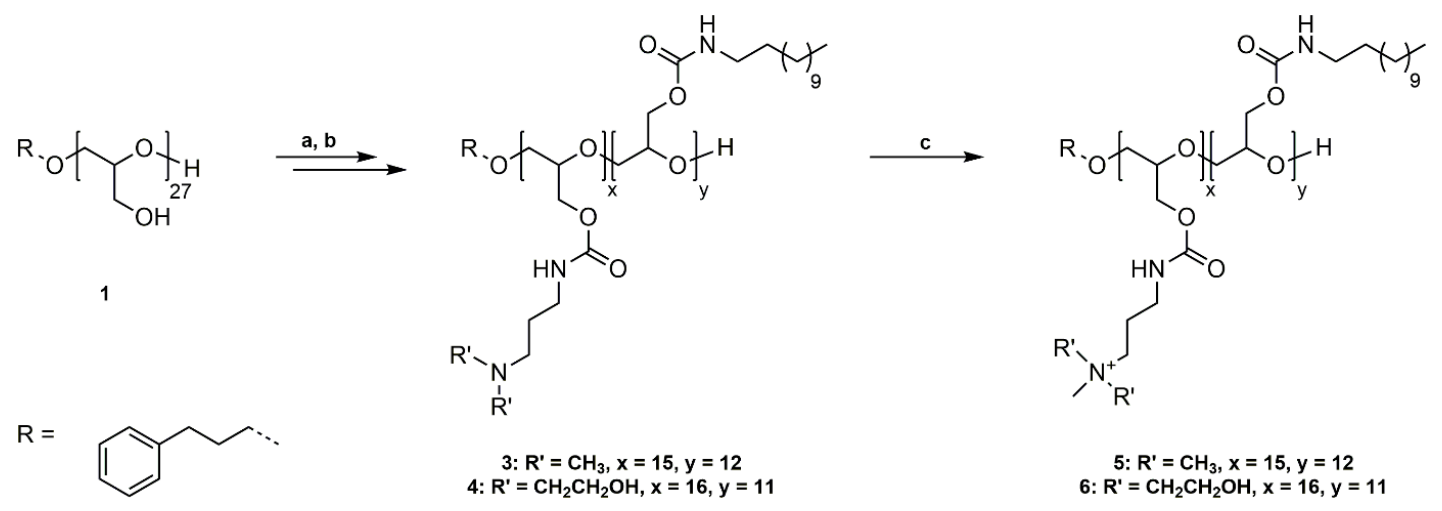

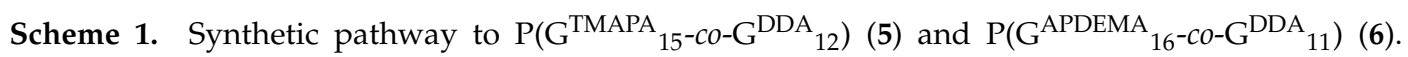
(a) Functionalization of $\mathrm{PG}_{27}$ (1) with phenyl chloroformate, pyridine/DCM, rt, $20 \mathrm{~h}$; (b) Reaction of $\mathrm{P}\left(\mathrm{G}^{\mathrm{PC}}\right)_{27}$ (2) with DDA and DMAPA/APDEA, THF, rt, $42 \mathrm{~h}$; (c) Quaternization of tertiary amines with methyl iodide, THF, rt, $20 \mathrm{~h}$.

${ }^{1} \mathrm{H}$ and ${ }^{13} \mathrm{C}$ NMR spectra of $\mathrm{P}\left(\mathrm{G}^{\mathrm{TMAPA}}{ }_{15-\mathrm{co}-\mathrm{G}^{\mathrm{DDA}}}{ }_{12}\right)(5)$ in DMSO- $d_{6}$ show characteristic signals of the dodecyl and trimethylpropylammonium groups adjacent to the carbamate moieties, proving the successful functionalization of $\mathrm{PG}_{27}$ (1). In the ${ }^{1} \mathrm{H}$ NMR spectrum (Figure 2a), the characteristic signals of the dodecyl functionality appear as three multiplets at $\delta=0.74-0.90$ (Signal 20), $\delta=1.30-1.44$ (Signal 18), and $\delta=2.85-2.99 \mathrm{ppm}$ (Signal 17), and a singlet at $\delta=1.20 \mathrm{ppm}$ (Signal 19). The trimethylpropylammonium groups are shown as three multiplets $\delta=1.72-1.91$ (Signal 11), $\delta=3.00-3.17$ (Signal 10/13), and $\delta=3.26-3.43 \mathrm{ppm}$ (Signal 12). A multiplet at $\delta=3.82-4.19 \mathrm{ppm}$ (Signal 9/16) shows the methylene group of the glycidol repeating unit. In the ${ }^{13} \mathrm{C}$ NMR spectrum (Figure S13), the distinctive signals of the dodecyl groups are found at $\delta=13.8$ (Signal 23), $\delta=22.0-28.9$ (Signal 22), and $\delta=31.2 \mathrm{ppm}$ (Signal 21). The representative signals of the trimethylpropylammonium functionality are shown at $\delta=26.2$ (Signal 13), $\delta=37.3$ (Signal 12), $\delta=52.2$ (Signal 15), and $\delta=63.2 \mathrm{ppm}$ (Signal 14). Further, the signal of the carbamate groups can be found at $\delta=156.0 \mathrm{ppm}$ (Signal 11/19). 


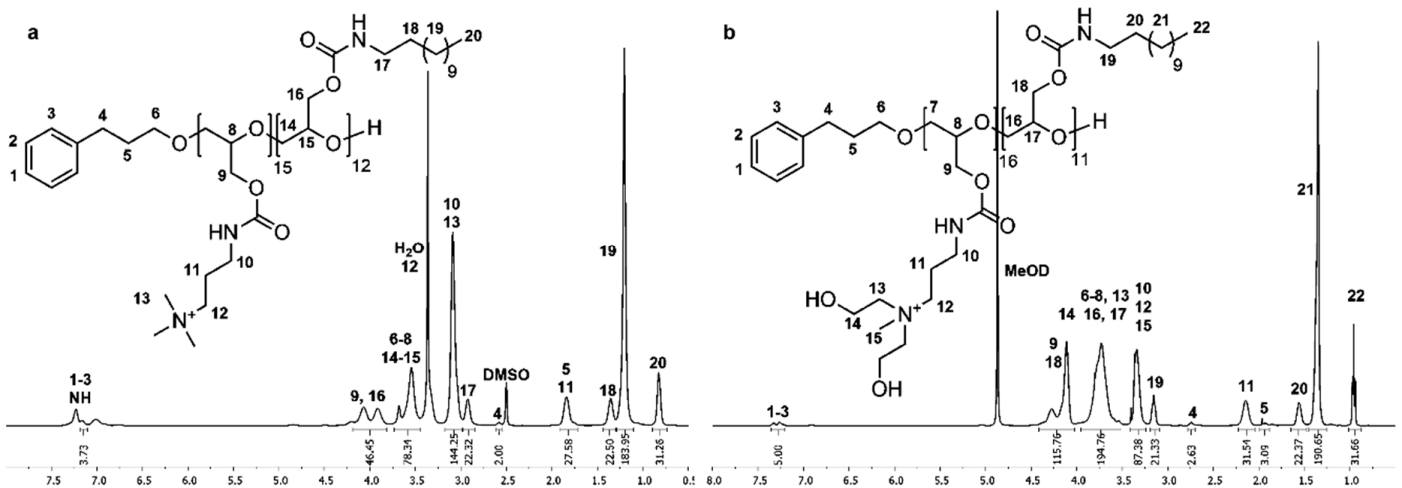

Figure 2. ${ }^{1} \mathrm{H}$ NMR spectra of $\mathrm{P}\left(\mathrm{G}^{\mathrm{TMAPA}}{ }_{15}-c 0-\mathrm{G}^{\mathrm{DDA}}{ }_{12}\right)$ (5) measured in DMSO- $d_{6}$ (a) and $\mathrm{P}\left(\mathrm{G}^{\mathrm{APDEMA}}{ }_{16}-\mathrm{Co}-\mathrm{G}^{\mathrm{DDA}}{ }_{11}\right)(\mathbf{6})$ measured in $\mathrm{MeOD}(\mathbf{b})$.

The number of DMAPA and DDA groups attached to $\mathrm{PG}_{27}(\mathbf{1})$ was calculated by comparing the signal intensity of one methylene group of the 3-phenyl-1-propanol (Signal 4) used in the synthesis of 1 with signals 10/11/13 and signals 18-20, respectively. The absolute molecular weight $\left(M_{n, N M R}\right)$ was calculated using the 3-phenyl-1-propyl end group as an internal reference $\left(M_{\mathrm{n}, \mathrm{NMR}}=10,111 \mathrm{~g} \cdot \mathrm{mol}^{-1}\right)$. Full functionalization of $\mathrm{PG}_{27}$ (1) was reached.

SEC analysis using DMF as eluent confirms the synthesis of $\mathrm{P}\left(\mathrm{G}^{\mathrm{TMAPA}}{ }_{15}-\mathrm{co}-\mathrm{G}^{\mathrm{DDA}}{ }_{12}\right)$ (5) with $M_{\mathrm{n}, \mathrm{SEC}}=4400 \mathrm{~g} \cdot \mathrm{mol}^{-1}$ and a molecular weight distribution of $\emptyset=1.18$ (Figure S14). However, due to the amphiphilic nature of this polymer and resulting interactions with the column, the molecular weight measured by SEC analysis is lower than the molecular weight calculated from the ${ }^{1} \mathrm{H}$ NMR spectrum.

${ }^{1} \mathrm{H}$ and ${ }^{13} \mathrm{C}$ NMR spectra of $\mathrm{P}\left(\mathrm{G}^{\mathrm{APDEMA}}{ }_{16}-\mathrm{Co}-\mathrm{G}^{\mathrm{DDA}}{ }_{11}\right)(6)$ were measured in MeOD due to the poor solubility of the polymer in DMSO- $d_{6}$. In the ${ }^{1} \mathrm{H}$ NMR spectrum (Figure $2 b$ ), this leads to a shift of all signals to lower field. Thus, the characteristic signals for the dodecyl functionality and the cationic moiety can be found in analogy to $\mathrm{P}\left(\mathrm{G}^{\mathrm{TMAPA}}{ }_{15}-\mathrm{co}-\mathrm{G}^{\mathrm{DDA}}{ }_{12}\right)$ (5). Additionally, the signals of the hydroxyethyl group can be found in the multiplets at $\delta=3.51-3.96$ (Signal 13) and $\delta=4.02-4.42 \mathrm{ppm}$ (Signal 14). In the ${ }^{13} \mathrm{C}$ NMR spectrum (Figure S15), the signals are also shifted to lower fields in comparison with $\mathrm{P}\left(\mathrm{G}^{\mathrm{TMAPA}}{ }_{15}-\mathrm{co}-\mathrm{G}^{\mathrm{DDA}}{ }_{12}\right)(5)$, except for signals of the methyl group and methylene group adjacent to the cationic moiety that are shifted to higher fields. The methyl group can be found at $\delta=50.9 \mathrm{ppm}$ (Signal 17) and the methylene group is found at $\delta=56.8 \mathrm{ppm}$ (Signal 14). Additionally, the hydroxyethyl group is shown at $\delta=65.4 \mathrm{ppm}$ (Signal 15/16). The number of functionalities and the absolute molecular weight was calculated as described previously $\left(\mathrm{M}_{\mathrm{n} . \mathrm{NMR}}=9608 \mathrm{~g} \cdot \mathrm{mol}^{-1}\right)$. Both spectra confirm the successful synthesis of $\mathrm{P}\left(\mathrm{G}^{\mathrm{APDEMA}}{ }_{16}-\mathrm{CO}-\mathrm{G}^{\mathrm{DDA}}{ }_{11}\right)$ (6).

SEC analysis of $\mathrm{P}\left(\mathrm{G}^{\mathrm{APDEMA}}{ }_{16}-\mathrm{Co}-\mathrm{G}^{\mathrm{DDA}}{ }_{11}\right)(6)$ using DMF was not possible. SEC analysis of the precursor $\mathrm{P}\left(\mathrm{G}^{\mathrm{APDEA}}{ }_{16}-\mathrm{co}-\mathrm{G}^{\mathrm{DDA}}{ }_{11}\right)(4)$ confirmed the successful synthesis with $M_{\mathrm{n}, \mathrm{SEC}}=14,100 \mathrm{~g} \cdot \mathrm{mol}^{-1}$ and a molecular weight distribution of $Đ=3.56$ (Figure S12). However, hydrogen bonding between polymer molecules leads to the generation of polymer aggregates and, thus, a high molecular weight and a broad distribution.

\subsection{Synthesis of $P\left(G^{D D A c, q}\right)_{27}(\mathbf{1 0})$}

The previous part showed that phenyl carbonates are excellent electrophiles for the substitution reaction of non-functionalized, reactive, primary amines. For the reaction with homocysteine thiolactone the electrophilicity of the carbonate needs to be higher than that of the carbonyl group of the thiolactone ring to prevent the reaction of the homocysteine thiolactone with itself. We have shown in our previous work that 4-nitrophenyl carbonates show a higher reactivity compared to phenyl carbonates in aminolysis reactions and are suitable for the reaction with functional, primary amines $[41,42]$. 
$\mathrm{PG}_{27}$ (1) was reacted with 4-nitrophenyl chloroformate in pyridine/dichloromethane at room temperature (Scheme 2a). For purification, poly(glycidyl 4-nitrophenyl carbonate) ${ }_{27}(7)$ was washed with water, $1 \mathrm{M} \mathrm{HCl}$ solution (aq.), and saturated $\mathrm{NaCl}$ solution (aq.) to remove pyridine hydrochloride and excess pyridine, and precipitated in methanol. The successful functionalization was confirmed by ${ }^{1} \mathrm{H},{ }^{13} \mathrm{C}$ NMR spectroscopy, and SEC analysis (Figures S16-S18). P(G $\left(\mathrm{G}^{\mathrm{NPC}}\right)_{27}$ was afterwards reacted with DL-homocysteine thiolactone hydrochloride in DMF at room temperature using catalytic amounts of 4-DMAP and $\mathrm{Et}_{3} \mathrm{~N}$ as a base (Scheme $2 \mathrm{~b}$ ). The resulting poly(glycidyl homocysteine thiolactonylcarbamate $)_{27}\left(\mathrm{P}\left(\mathrm{G}^{\mathrm{HCTL}}\right)_{27}\right)(8)$ was purified by precipitation in methanol and characterized by ${ }^{1} \mathrm{H},{ }^{13} \mathrm{C}$ NMR spectroscopy, and SEC analysis (Figures S19-S21). In a one-pot reaction, $\mathrm{P}\left(\mathrm{G}^{\mathrm{HCTL}}\right)_{27}$ (8) was reacted with DMAPA under ring-opening in $\mathrm{CHCl}_{3}$ at room temperature, and the generated thiol was converted with dodecyl acrylate in a thiol-ene reaction. The synthesized $\mathrm{P}\left(G^{\mathrm{DDAc}}\right)_{27}$ (9) was purified by dialysis in acetone and characterized by ${ }^{1} \mathrm{H}$ NMR, ${ }^{13} \mathrm{C}$ NMR spectroscopy, and SEC analysis (Figures S22-S24). $\mathrm{P}\left(\mathrm{G}^{\mathrm{DDAc}}\right)_{27}$ (9) was subsequently quaternized with methyl iodide in THF at room temperature. The crude product was dialyzed in methanol to remove excess methyl iodide, and $\mathrm{P}\left(\mathrm{G}^{\mathrm{DDAc}, \mathrm{q}}\right)(\mathbf{1 0})$ was described by ${ }^{1} \mathrm{H},{ }^{13} \mathrm{C}$ NMR spectroscopy, and SEC analysis.

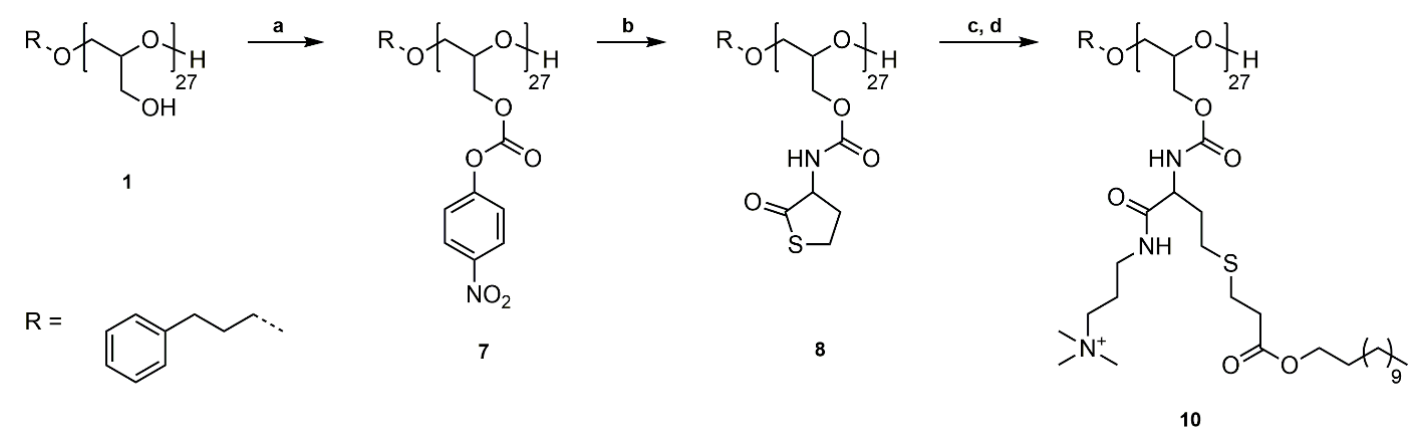

Scheme 2. Synthetic pathway to $\mathrm{P}\left(\mathrm{G}^{\mathrm{DDAc}} \mathrm{q}\right)_{27}$ (10). (a) Functionalization of $\mathrm{PG}_{27}$ (1) with 4-nitrophenyl chloroformate, pyridine/DCM, rt, 20 h; (b) Reaction of $\mathrm{P}\left(\mathrm{G}^{\mathrm{NPC}}\right)_{27}$ (7) with DL-homocysteine thiolactone hydrochloride, 4-DMAP, $\mathrm{Et}_{3} \mathrm{~N}$, DMF, rt, 20 h; (c) Ring-opening reaction with DMAPA, followed by thiol-ene reaction with dodecyl acrylate, $\mathrm{CHCl}_{3}, \mathrm{rt}, 20 \mathrm{~h}$; (d) Quaternization of tertiary amines with methyl iodide, THF, rt, $20 \mathrm{~h}$.

$P\left(G^{D D A c}, q\right)_{27}$ (10) exhibits characteristic signals of the introduced functionalities in the ${ }^{1} \mathrm{H}$ and ${ }^{13} \mathrm{C}$ NMR spectra measured in DMSO- $d_{6}$. In the ${ }^{1} \mathrm{H}$ NMR spectrum (Figure 3a), the trimethylpropylammonium group is described by two multiplets at $\delta=1.85-2.25$ (Signal 13) and $\delta=3.05-3.50 \mathrm{ppm}$ (Signals 12/14/15). The dodecyl acrylate moiety is shown as a triplet at $\delta=0.81 \mathrm{ppm}$ (Signal 23) and two multiplets at $\delta=1.05-1.32$ (Signal 22) and $\delta=1.46-1.62 \mathrm{ppm}$ (Signal 21). Two multiplets at $\delta=2.39-2.64$ (Signals 17/19) and $\delta=2.66-2.82 \mathrm{ppm}$ (Signal 18) are characteristic for the methylene groups adjacent to the thioether. The signals at $\delta=3.84-4.50 \mathrm{ppm}$ (Signals 10/11/20) show the methylene groups adjacent to the ester functionality and the single proton adjacent to the carbamate moiety. The multiplet also contains the methylene groups of the polyglycidol repeating unit adjacent to the carbamate. In the ${ }^{13} \mathrm{C}$ NMR spectrum (Figure S25), the distinctive signals can be found at $\delta=28.3$ (Signal 15), $\delta=40.1$ (Signal 14), $\delta=53.8$ (Signal 17 ), and $\delta=64.9 \mathrm{ppm}$ (Signal 16) for the trimethylpropylammonium functionality; at $\delta=14.1$ (Signal 26), $\delta=22.6-31.9$ (Signals 24/25), and $\delta=64.9 \mathrm{ppm}$ (Signal 23) for the dodecyl acrylate; and at $\delta=26.7, \delta=28.6$ (Signal 18-20) and $\delta=34.8 \mathrm{ppm}$ (Signal 21) for the methylene groups adjacent to the thioether. The number of functionalities and the absolute molecular weight was calculated as described previously $\left(M_{\mathrm{n} . \mathrm{NMR}}=18,947 \mathrm{~g} \cdot \mathrm{mol}^{-1}\right)$. Both spectra confirm the successful synthesis of $P\left(G^{D D A c}, q\right)(\mathbf{1 0})$. 


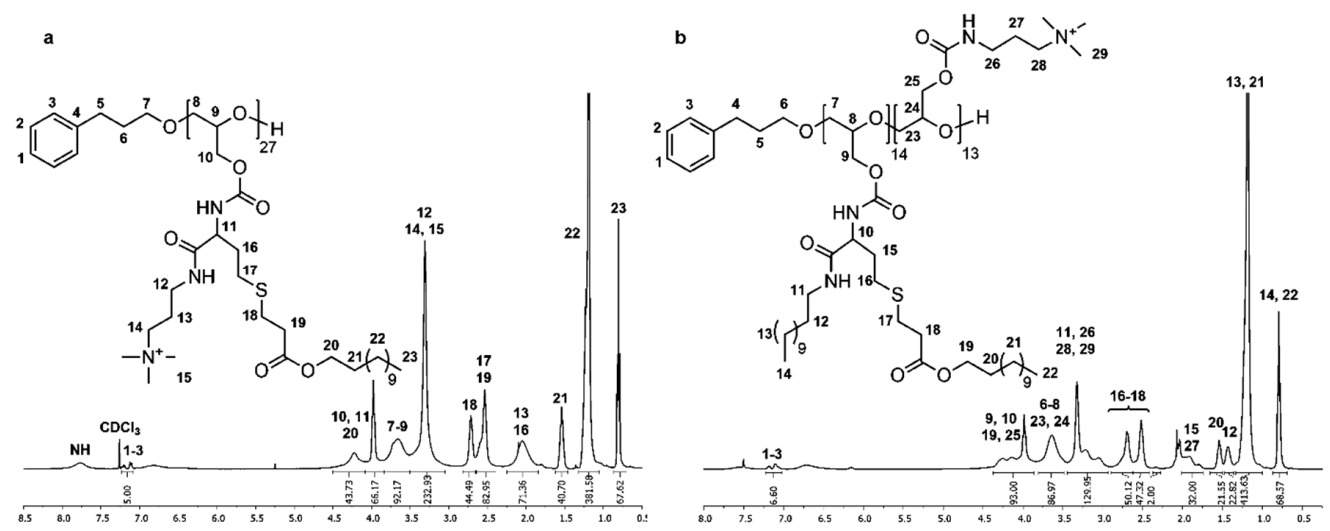

Figure 3. ${ }^{1} \mathrm{H}$ NMR spectra of $\mathrm{P}\left(\mathrm{G}^{\mathrm{DDAc}}, \mathrm{q}\right)_{27}(\mathbf{1 0})$ measured in $\mathrm{CDCl}_{3}(\mathbf{a})$ and $\mathrm{P}\left(\mathrm{G}^{\mathrm{TMAPA}}{ }_{14}-\mathrm{co}-\mathrm{G}^{\mathrm{DDADDAc}}{ }_{13}\right)$ (12) measured in $\mathrm{CDCl}_{3}$ /acetone (6:4) (b).

SEC analysis using DMF as eluent also confirms the successful synthesis of $\mathrm{P}\left(\mathrm{G}^{\mathrm{DDAc}, \mathrm{q}}\right)_{27}$ (10) with $M_{\mathrm{n}, \mathrm{SEC}}=11,800 \mathrm{~g} \cdot \mathrm{mol}^{-1}$ and a molecular weight distribution of $\boxminus=1.36$ (Figure S26).

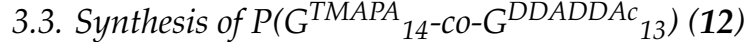

The preparation of $\mathrm{P}\left(\mathrm{G}^{\mathrm{TMAPA}}{ }_{14}-\mathrm{co}-\mathrm{G}^{\mathrm{DDADDAc}}{ }_{13}\right)$ (12) combines both previously described synthetic approaches. In a one-pot synthesis, $\mathrm{P}\left(\mathrm{G}^{\mathrm{NPC}}\right)_{27}(7)$ was first reacted with DMAPA in DMF at room temperature for $20 \mathrm{~h}$ (Scheme 3a). After full conversion of the primary amine (monitored by ${ }^{1} \mathrm{H}$ NMR spectroscopy), 4-DMAP, DL-homocysteine thiolactone hydrochloride, and triethylamine were added, and the reaction was stirred for another $20 \mathrm{~h}$ at room temperature. The crude product was reacted without further purification with dodecylamine and dodecyl acrylate in chloroform at room temperature (Scheme $3 b$ ). The crude product was purified by dialysis in acetone and $\mathrm{P}\left(\mathrm{G}^{\mathrm{DMAPA}}{ }_{14}\right.$-co-G $\left.^{\mathrm{DDADDAc}}{ }_{13}\right)(\mathbf{1 1})$ characterized by ${ }^{1} \mathrm{H}$ NMR, ${ }^{13} \mathrm{C}$ NMR spectroscopy, and SEC analysis (Figures S27-S29).

$\mathrm{P}\left(\mathrm{G}^{\mathrm{DMAPA}}{ }_{14}-\mathrm{co}-\mathrm{G}^{\mathrm{DDADDAc}}{ }_{13}\right.$ (11) was subsequently quaternized with methyl iodide in THF at room temperature, purified by dialysis in methanol, and characterized by the previously described methods (Scheme 3c).

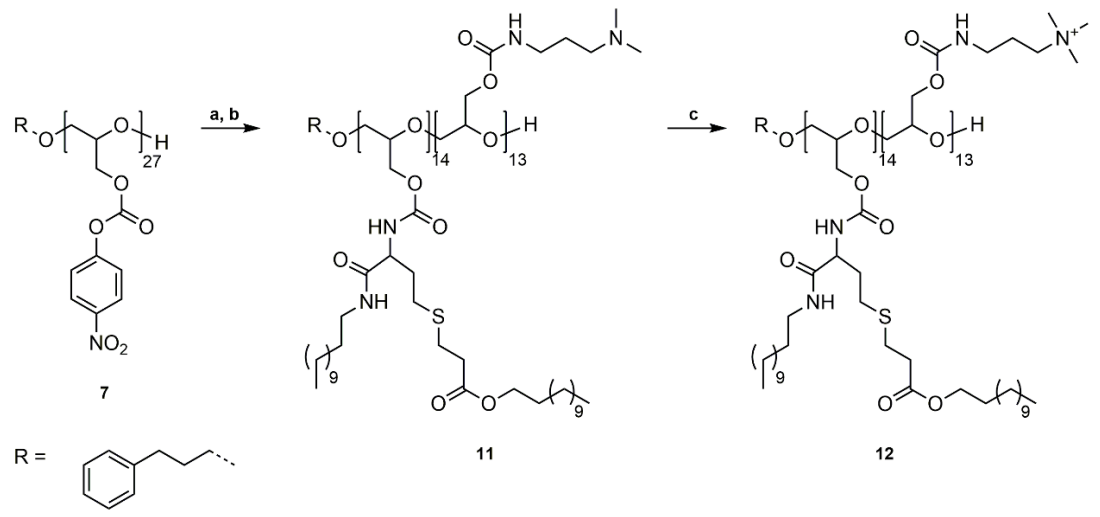

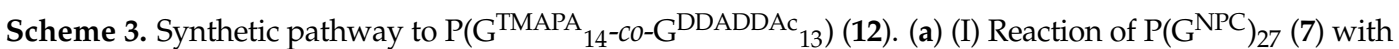
DMAPA, DMF, rt, 20 h, (II) Reaction with DL-homocysteine thiolactone hydrochloride, 4-DMAP, Et ${ }_{3} \mathrm{~N}$, DMF, 20 h; (b) Ring-opening reaction with dodecylamine, followed by thiol-ene reaction with dodecyl acrylate, $\mathrm{CHCl}_{3}, \mathrm{rt}, 20 \mathrm{~h}$; (c) Quaternization of tertiary amines with methyl iodide, THF, rt, $20 \mathrm{~h}$.

Due to the strong amphiphilic nature of $\mathrm{P}\left(\mathrm{G}^{\mathrm{TMAPA}}{ }_{14}-\mathrm{Co}-\mathrm{G}^{\mathrm{DDADDAc}}{ }_{13}\right)(\mathbf{1 2}),{ }^{1} \mathrm{H}$ and ${ }^{13} \mathrm{C} \mathrm{NMR}$ spectroscopy were performed in a mixture of $\mathrm{CDCl}_{3}$ and acetone- $d_{6}$. In the ${ }^{1} \mathrm{H}$ NMR spectrum 
(Figure $3 b$ ), the distinctive signals of the hydrophobic moiety are found at $\delta=0.80$ (Signal 14/22), $\delta=1.18$ (Signal 13/21) $\delta=1.36-1.48$ (Signal 12), and $\delta=1.50-1.66 \mathrm{ppm}$ (Signal 20). The methylene group adjacent to the ester is shown at $\delta=3.87-4.38 \mathrm{ppm}$ (Signal 19). This signal also includes the methylene groups (Signal 9/25) and the single proton (Signal 10) adjacent to the carbamate moieties. Specific signals of the cationic functionalities are found at $\delta=1.74-2.01$ (Signal 27) and $\delta=2.94-3.45 \mathrm{ppm}$ (Signal 26/28/29). The shift of the signals of all functional groups is in good comparison with the polymers described earlier. In the ${ }^{13} \mathrm{C}$ NMR spectrum, the shifts of the various signals are also in good comparison to the previously characterized cationic-hydrophobic polymers (Figure S30). Both spectra confirm the successful synthesis of $\mathrm{P}\left(\mathrm{G}^{\mathrm{TMAPA}}{ }_{14}-\mathrm{co}_{-}-\mathrm{G}^{\mathrm{DDADDAc}}{ }_{13}\right)$ (12).

The number of functionalities and the absolute molecular weight was calculated as described previously $\left(M_{\text {n.NMR }}=13,476 \mathrm{~g} \cdot \mathrm{mol}^{-1}\right)$. Although, the integral values shown in the ${ }^{1} \mathrm{H}$ NMR spectra are too low to be accurate for a polyether with 27 repeating units, the ratio of hydrophobic to cationic moieties is accepted as accurate. The absolute values of hydrophobic to cationic groups is extrapolated to match the expected number of repeating units. Fractionation towards lower molecular weights is excluded based on the method of purification.

SEC analysis using DMF as eluent also confirms the successful synthesis of $\mathrm{P}\left(\mathrm{G}^{\mathrm{TMAPA}}{ }_{14}-\mathrm{co}-\mathrm{G}^{\mathrm{DDADDAc}}{ }_{13}\right)(\mathbf{1 2})$ with $M_{\mathrm{n}, \mathrm{SEC}}=9400 \mathrm{~g} \cdot \mathrm{mol}^{-1}$ and a molecular weight distribution of $Ð=1.41$ (Figure S31).

\subsection{Antibacterial Efficacy}

To assess the influence of the microstructure of the functional polyglycidols on their antibacterial efficacy, the minimal inhibitory concentrations of the four synthesized polymers against E. coli and S. aureus were determined (Table 1).

Table 1. Minimal inhibitory concentration against E. coli and S. aureus and hemolytic activity of functional polyglycidols with defined microstructures $\mathrm{P}\left(\mathrm{G}^{\mathrm{TMAPA}}{ }_{15-\mathrm{co}-\mathrm{G}^{\mathrm{DDA}}}{ }_{12}\right)(5), \mathrm{P}\left(\mathrm{G}^{\mathrm{APDEMA}}{ }_{16}{ }^{-\mathrm{co}-}\right.$ $\left.G^{\mathrm{DDA}}{ }_{11}\right)(\mathbf{6}), \mathrm{P}\left(\mathrm{G}^{\mathrm{DDAc}, \mathrm{q}}\right)_{27}(\mathbf{1 0}), \mathrm{P}\left(\mathrm{G}^{\mathrm{TMAPA}}{ }_{14}-\mathrm{co}-\mathrm{G}^{\mathrm{DDADDAc}}{ }_{13}\right)$ (12).

\begin{tabular}{|c|c|c|c|c|c|}
\hline \multirow[b]{2}{*}{ Polymer } & \multirow[b]{2}{*}{ Microstructure } & \multirow[t]{2}{*}{$M_{\mathrm{n}, \mathrm{NMR}}{ }^{\mathrm{a}}\left(\mathrm{g} \cdot \mathrm{mol}^{-1}\right)$} & \multicolumn{2}{|c|}{$\mathrm{MIC}_{100}{ }^{\mathrm{b}, \mathrm{c}}(\mu \mathrm{g} / \mathrm{mL})$} & \multirow[t]{2}{*}{$\begin{array}{l}\mathrm{HC}_{50}{ }^{\mathrm{d}} \\
(\mu \mathrm{g} / \mathrm{mL})\end{array}$} \\
\hline & & & E. coli & S. aureus & \\
\hline 5 & $\mathrm{P}\left(\mathrm{G}^{\mathrm{TMAPA}}{ }_{15}-\mathrm{co}-\mathrm{G}^{\mathrm{DDA}}{ }_{12}\right)$ & 10,111 & 30 & 20 & $>>500^{f}$ \\
\hline 6 & $\mathrm{P}\left(\mathrm{G}^{\mathrm{APDEMA}}{ }_{16-\mathrm{Co}-\mathrm{G}^{\mathrm{DDA}}}{ }_{11}\right)$ & 9608 & 30 & 30 & 100 \\
\hline 10 & $P\left(G^{\text {DDAc, }}\right)_{27}$ & 18,947 & 30 & $>200$ & $<10$ \\
\hline 12 & $\mathrm{P}\left(\mathrm{G}^{\mathrm{TMAPA}}{ }_{14}-c 0-\mathrm{G}^{\mathrm{DDADDAc}}{ }_{13}\right)$ & 13,476 & $200^{d}$ & $>200-500^{\mathrm{e}}$ & $<10$ \\
\hline
\end{tabular}

${ }^{a}$ Molecular weight $\left(\mathrm{M}_{\mathrm{n}, \mathrm{NMR}}\right)$ calculated from ${ }^{1} \mathrm{H}$ NMR with an accuracy of integration of $\pm 5 \%$; ${ }^{\mathrm{b}}$ Minimum inhibitory concentration which prevents the visible growth of $100 \%$ of the bacteria within $20 \mathrm{~h}$ monitoring time; ${ }^{\mathrm{c}}$ Inoculum size $=1 \times 10^{6}-2 \times 10^{6} \mathrm{CFU} \cdot \mathrm{mL}^{-1}$; ${ }^{\mathrm{d}} \mathrm{HC}_{50}$ : concentration causing $50 \%$ lysis (hemolysis) of red blood cells (RBCs) relative to Triton X-100 (positive control, $100 \%$ ), linear polyglycidol $\mathrm{PG}_{27}\left(\right.$ 1) $\mathrm{HC}_{50}>500 \mu \mathrm{g} / \mathrm{mL}$; ${ }^{\mathrm{e}}$ Non-growth value due to turbidity of solution caused by the polymer; ${ }^{\mathrm{f}} 10-500 \mu \mathrm{g} / \mathrm{mL}$ of polymer 5 causing agglutination of RBC, and about $6 \%$ (at $10 \mu \mathrm{g} / \mathrm{mL})-40 \%$ (at $500 \mu \mathrm{g} / \mathrm{mL}$ ) hemolysis.

In comparison to the other three polymers $\left(\mathbf{6}, \mathbf{1 0}\right.$, and 12) $\mathrm{P}\left(\mathrm{G}^{\mathrm{TMAPA}}{ }_{15}-c 0-\mathrm{G}^{\mathrm{DDA}}{ }_{12}\right)$ (5) —with cationic and hydrophobic groups statistically distributed at the polyglycidol backbone-exhibited the lowest MIC against both E. coli and S. aureus. A hydrophilic functionalization at the quaternary amine $\left(\mathrm{P}\left(\mathrm{G}^{\mathrm{APDEMA}}{ }_{16}-\mathrm{co}-\mathrm{G}^{\mathrm{DDA}}{ }_{11}\right)(6)\right)$ does not affect the efficacy against $E$. coli, but slightly impairs the efficacy against $S$. aureus.

When the cationic and the hydrophobic residues are located at every repeating unit of the polymer and are connected by a short spacer $\left(\mathrm{P}\left(\mathrm{G}^{\mathrm{DDAc}, \mathrm{q}}\right)_{27}(\mathbf{1 0})\right)$, the efficacy against $E$. coli remains the same as for $\mathbf{5}$ and $\mathbf{6}$. However, the efficacy against $S$. aureus decreases significantly by a factor of 10. The molecular weight of polymer $\mathbf{1 0}$ is approximately twice as high as the molecular weight of polymers $\mathbf{5}$ and $\mathbf{6}$. Thus, the higher MIC values in the case of S. aureus compared to E. coli might result 
from a sieving effect by the thick peptidoglycan layer of the Gram-positive bacterium. This was also discussed by Lienkamp et al. [43].

In polymer 10, the hydrophobic and cationic residues are connected by a spacer and are not directly linked to each other. Opposite to the resulting antibacterial effect of the latter (polymer 10), functionalized branched poly(ethylene imine)s (PEI) with amphiphilic grafts had better antibacterial properties against $S$. aureus than PEI with randomly linked cationic and hydrophobic grafts. However, best results were obtained with the cationic residue directly linked to the aliphatic residue without a spacer in between [30]. Thus, an influence of the kind of polymer backbone on the polymer architecture and of the kind of amphiphilic functionality on the antibacterial effect can be discussed.

Enhancing the hydrophobic share in comparison to the cationic functionality $\left(\mathrm{P}\left(\mathrm{G}^{\mathrm{TMAPA}}{ }_{14}-\mathrm{Co}-\mathrm{G}^{\mathrm{DDADDAc}}{ }_{13}\right)(\mathbf{1 2})\right.$ with two hydrophobic residues in the same polymer segment, connected by a spacer) leads to a decrease of efficacy by a factor of 10 for both E. coli and S. aureus. The high amount of hydrophobic residues induces aggregation in aqueous media, which might lead to a partial unavailability of functional groups of the polymer. Thus, interaction with the bacterial cell envelope is impaired, since a certain amount of cationic charge is necessary to initiate the binding to the bacterial cell envelope as the first step in the cascade of amphipathic polymers' mode of action.

Compared to the 1:2 ratio of cationic to hydrophobic residues in polymer 12, branched PEI with functionalities introduced via carbonate coupler chemistry and a higher ratio of cationic to hydrophobic residues resulted in higher efficacies in the case of E. coli [29]. Furthermore, azetidinium functionalized poly(vinylamine)s with a higher ratio of hydrophobic to cationic moieties resulted in better antibacterial efficacies against E. coli and against S. aureus [44]. Thus, apparently there is a strong influence of the polymer backbone and the structure of the cationic and hydrophobic side chains on the microstructure and the efficacy besides the hydrophilic to lipophilic balance of the respective polymer.

Additionally, polymers functionalized with amphiphilic couplers where the cationic moiety was directly linked to the hydrophobic residue exhibited the lowest MIC compared to randomly distributed cationic and hydrophobic groups linked via a spacer. Ganewatta et al. refer to a "same-centered" repeat unit structure with the hydrophobic moiety being directly accompanied by the charged moiety, i.e., the functional groups not being spatially separated over the backbone, leading to an amphiphilic balance at the monomer level [45].

\subsection{Hemolytic Activity}

To understand the selectivity of polymers $5, \mathbf{6}, \mathbf{1 0}$, and $\mathbf{1 2}$ to affect bacterial cells and mammalian cells, a hemolysis test was performed, and the concentration required for $50 \%$ lysis of human red blood cells RBCs was determined ( $\mathrm{HC}_{50}$, Table 1). Linear polyglycidol $\mathrm{PG}_{27}(\mathbf{1})$ did not show any significant lysis of RBCs even at the highest concentration $(500 \mu \mathrm{g} / \mathrm{mL})$ tested. For polymer 5 at the concentrations tested $(10-500 \mu \mathrm{g} / \mathrm{mL})$, no $\mathrm{HC}_{50}$ value could be given at the tested concentrations $(10-500 \mu \mathrm{g} / \mathrm{mL})$. Hemolysis amounted to about $6 \%$ at $10 \mu \mathrm{g} / \mathrm{mL}, 30 \%$ at $30 \mu \mathrm{g} / \mathrm{mL}$, and $40 \%$ at $500 \mu \mathrm{g} / \mathrm{mL}$. However, agglutination of RBCs occurred at tested concentrations. $\mathrm{HC}_{50}$ value for polymer $\mathbf{6}$ amounted to $100 \mu \mathrm{g} / \mathrm{mL}$. The higher value of $\mathrm{HC}_{50}$ compared to the $\mathrm{MIC}_{100}$ against $E$. coli and $S$. aureus $(30 \mu \mathrm{g} / \mathrm{mL})$ proved that polymer $\mathbf{6}$ has a selectivity to differentiate between mammalian cells and bacterial cell walls. For comparison, the $\mathrm{HC}_{50}$ values of polymers $\mathbf{1 0}$ and $\mathbf{1 2}$ were below the lowest concentration $(10 \mu \mathrm{g} / \mathrm{mL})$ tested, i.e., had a low selectivity for bacterial cell envelopes.

\section{Conclusions}

We successfully synthesized various novel cationic-hydrophobic functionalized polyglycidols. A polyglycidol with statistically distributed cationic and hydrophobic groups (cationic-hydrophobic ratio of 1:1), a polyglycidol with a hydrophilic modification at the cationic moieties, a polyglycidol with cationic and hydrophobic functionalities at every repeating unit, and a polyglycidol with a cationic-hydrophobic balance of 1:2 were characterized by ${ }^{1} \mathrm{H}$ NMR, ${ }^{13} \mathrm{C}$ NMR spectroscopy, and SEC analyses and evaluated in regard to their antibacterial activity. 
Antibacterial polyglycidols with statistic distribution of cationic and hydrophobic residues are equally active against a Gram-negative and a Gram-positive bacterial strain. In contrast to this, the efficacy of amphipathic poly(ethylene imine)s is higher by a factor of 10 against Gram-positive bacteria than against Gram-negative ones. However, antibacterial polyglycidols are equally or better active against $E$. coli than against $S$. aureus. The best efficacy against both bacterial strains resulted from the polyglycidol with statistic distribution of the cationic and hydrophobic residues with polymer 6, showing also the best selectivity to differentiate between mammalian cells and bacterial cell walls compared with the other polymers tested in this study.

When cationic and hydrophobic residues are located in the same polyglycidol repeating unit being connected by a spacer, the impact on the cell envelope of $S$. aureus is significantly less effective than against $E$. coli. The outer part of the cell envelope of Gram-positive bacteria is composed of a thick peptidoglycan layer with integrated lipoteichoic acids followed by the inner cell membrane. Here, a sieving effect might be discussed, retarding the interaction of this polymer with the underlying cell membrane. Additionally, the vicinity of the cationic and hydrophobic groups in these polyglycidols compared to those prepared with statistic distribution of cationic and hydrophobic residues might disturb the interaction cascade of amphipathic polymers with the components of the cell envelope.

Changing the hydrophilic-lipophilic balance (HLB) to a higher lipophilic-cationic ratio in amphipathic polyglycidol with statistically distributed cationic and hydrophobic residues leads to a decrease in efficacy in the same order of magnitude against both bacterial strains E. coli and S. aureus. Antimicrobial efficacy of an amphipathic polymer strongly depends on its HLB, the microstructure induced by the type of polymer backbone, and the distribution and kind of the cationic and hydrophobic functionalities.

Supplementary Materials: The following are available online at www.mdpi.com/2073-4360/10/1/96/s1, Figure S1: ${ }^{1} \mathrm{H}$ NMR spectrum of $\mathrm{PG}_{27}(\mathbf{1})$ measured in DMSO- $d_{6}$, Figure S2: ${ }^{13} \mathrm{C}$ NMR spectrum of $\mathrm{PG}_{27}$ (1) measured in DMSO- $d_{6}$, Figure S3: DMF-SEC traces of $\mathrm{PG}_{27}(\mathbf{1})$, Scheme S1: Synthetic pathway to P(G $\left(G^{\mathrm{TMAPA}}{ }_{15}-\mathrm{co}-\mathrm{G}^{\mathrm{DDA}}{ }_{12}\right)$

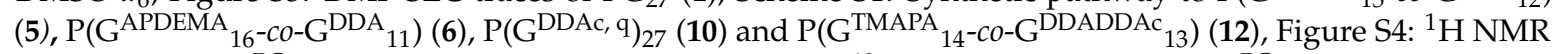
spectrum of $\mathrm{P}\left(\mathrm{G}^{\mathrm{PC}}\right)_{27}$ (2) measured in $\mathrm{CDCl}_{3}$, Figure $\mathrm{S} 5:{ }^{13} \mathrm{C}$ NMR spectrum of $\mathrm{P}\left(\mathrm{G}^{\mathrm{PC}}\right)_{27}$ (2) measured in $\mathrm{CDCl}_{3}$, Figure S6: DMF-SEC traces of $\mathrm{P}\left(\mathrm{G}^{\mathrm{PC}}\right)_{27}(2)$, Figure S7: ${ }^{1} \mathrm{H}$ NMR spectrum of $\mathrm{P}\left(\mathrm{G}^{\mathrm{DMAPA}}{ }_{15}-\mathrm{co}-\mathrm{G}^{\mathrm{DDA}}{ }_{12}\right)(3)$ measured in $\mathrm{CDCl}_{3}$, Figure S8: ${ }^{13} \mathrm{C}$ NMR spectrum of $\mathrm{P}\left(\mathrm{G}^{\mathrm{DMAPA}}{ }_{15}-\mathrm{co}-\mathrm{G}^{\mathrm{DDA}}{ }_{12}\right)(3)$ measured in $\mathrm{CDCl}_{3}$, Figure S9: DMF-SEC traces of P(G (GMAPA $\left.15^{-c o-G} \mathrm{G}^{\mathrm{DDA}}{ }_{12}\right)(3)$, Figure S10: ${ }^{1} \mathrm{H}$ NMR spectrum of $\mathrm{P}\left(\mathrm{G}^{\mathrm{APDEA}}{ }_{16}\right.$-co- $\left.^{\mathrm{DDDA}}{ }_{11}\right)$ (4) measured in MeOD, Figure S11: ${ }^{13} \mathrm{C}$ NMR spectrum of $\mathrm{P}\left(\mathrm{G}^{\mathrm{APDEA}}{ }_{16}-\mathrm{Co}-\mathrm{G}^{\mathrm{DDA}}{ }_{11}\right)$ (4) measured in MeOD, Figure

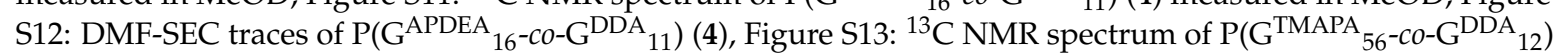
(5) measured in DMSO- $d_{6}$, Figure S14: DMF-SEC traces of $\mathrm{P}\left(\mathrm{G}^{\mathrm{TMAPA}}{ }_{56^{-}-\mathrm{Co}-\mathrm{G}^{\mathrm{DDA}}}{ }_{12}\right)(5)$, Figure S15: ${ }^{13} \mathrm{C}$ NMR spectrum of $\mathrm{P}\left(\mathrm{G}^{\mathrm{APDEMA}}{ }_{16}-\mathrm{co}-\mathrm{G}^{\mathrm{DDA}}{ }_{11}\right)(6)$ measured in MeOD, Figure $\mathrm{S} 16:{ }^{1} \mathrm{H}$ NMR spectrum of $\mathrm{P}\left(\mathrm{G}^{\mathrm{NPC}}\right)_{27}(7)$ measured in DMSO- $d_{6}$, Figure S17: ${ }^{13} \mathrm{C}$ NMR spectrum of $\mathrm{P}\left(\mathrm{G}^{\mathrm{NPC}}\right)_{27}(7)$ measured in DMSO- $d_{6}$, Figure S18: DMF-SEC traces of $\mathrm{P}\left(\mathrm{G}^{\mathrm{NPC}}\right)_{27}(7)$, Figure S19: ${ }^{1} \mathrm{H}$ NMR spectrum of $\mathrm{P}\left(\mathrm{G}^{\mathrm{HCTL}}\right)_{27}(8)$ measured in DMSO- $d_{6}$, Figure S20: ${ }^{13} \mathrm{C}$ NMR spectrum of $\mathrm{P}\left(\mathrm{G}^{\mathrm{HCTL}}\right)_{27}(8)$ measured in DMSO- $d_{6}$, Figure S21: DMF-SEC traces of $\mathrm{P}\left(\mathrm{G}^{\mathrm{HCTL}}\right)_{27}$ (8), Figure S22: ${ }^{1} \mathrm{H}$ NMR spectrum of $\mathrm{P}\left(\mathrm{G}^{\mathrm{DDAc}}\right)_{27}(9)$ measured in $\mathrm{CDCl}_{3}$. Figure S23: ${ }^{13} \mathrm{C}$ NMR spectrum of $\mathrm{P}\left(\mathrm{G}^{\mathrm{DDAc}}\right)_{27}(9)$ measured in $\mathrm{CDCl}_{3}$, Figure S24: DMF-SEC traces of $\mathrm{P}\left(\mathrm{G}^{\mathrm{DDAc}}\right)_{27}(9)$, Figure S25: ${ }^{13} \mathrm{C}$ NMR spectrum of $\mathrm{P}\left(\mathrm{G}^{\mathrm{DDAc}, \mathrm{q}}\right)_{27}(\mathbf{1 0})$ measured in $\mathrm{CDCl}_{3}$, Figure S26: DMF-SEC traces of $\mathrm{P}\left(\mathrm{G}^{\mathrm{DDAc}, \mathrm{q}}\right)_{27}(\mathbf{1 0})$, Figure S27: ${ }^{1} \mathrm{H}$ NMR spectrum of $\mathrm{P}\left(\mathrm{G}^{\mathrm{DMAPA}}{ }_{14}-\mathrm{co}-\mathrm{G}^{\mathrm{DDADDAc}}{ }_{13}\right)(\mathbf{1 1})$ measured in $\mathrm{CDCl}_{3} /$ acetone- $d_{6}$, Figure S28: ${ }^{13} \mathrm{C}$ NMR spectrum of $\mathrm{P}\left(\mathrm{G}^{\mathrm{DMAPA}}{ }_{14}-\mathrm{co}-\mathrm{G}^{\mathrm{DDADDAc}}{ }_{13}\right)(11)$ measured in $\mathrm{CDCl}_{3} /$ acetone- $d_{6}$, Figure S29: DMF-SEC traces of $\mathrm{P}\left(\mathrm{G}^{\mathrm{DMAPA}}{ }_{14}-\mathrm{co}-\mathrm{G}^{\mathrm{DDADDAc}}{ }_{13}\right)(\mathbf{1 1})$, Figure S30: ${ }^{13} \mathrm{C}$ NMR spectrum of $\mathrm{P}\left(\mathrm{G}^{\mathrm{TMAPA}} 14-c o-\mathrm{G}^{\mathrm{DDADDAc}}{ }_{13}\right)(\mathbf{1 2})$ measured in $\mathrm{CDCl}_{3} /$ acetone- $d_{6}$, Figure S31: DMF-SEC traces of P( $\left(\mathrm{G}^{\mathrm{TMAPA}}{ }_{14}-\mathrm{co}-\mathrm{G}^{\mathrm{DDADDAc}}{ }_{13}\right)(\mathbf{1 2})$.

Acknowledgments: We thank Rainer Haas for SEC analyses. This work was performed in part by the Center for Chemical Polymer Technology (CPT), which was supported by the EU and the federal state of North Rhine-Westphalia (Grant No. EFRE 3000883 02).

Author Contributions: The idea for this work comes from Helmut Keul and Martin Möller. Design of experiments and evaluation of results was performed by Fabian Marquardt, Cornelia Stöcker, and Helmut Keul. Antibacterial studies were performed, analyzed, and evaluated by Rita Gartzen and Elisabeth Heine. Presentation of the results and the structure of the manuscript were discussed with all authors; Fabian Marquardt wrote the first draft and included corrections suggested by the coauthors.

Conflicts of Interest: The authors declare no conflict of interest. 


\section{References}

1. Monteiro, D.R.; Gorup, L.F.; Takamiya, A.S.; Ruvollo-Filho, A.C.; de Camargo, E.R.; Barbosa, D.B. The growing importance of materials that prevent microbial adhesion: Antimicrobial effect of medical devices containing silver. Int. J. Antimicrob. Agents 2009, 34, 103-110. [CrossRef] [PubMed]

2. Spellberg, B.; Powers, J.H.; Brass, E.P.; Miller, L.G.; Edwards, J.E., Jr. Trends in antimicrobial drug development: Implications for the future. Clin. Infect. Dis. 2004, 38, 1279-1286. [CrossRef] [PubMed]

3. Li, Q.; Mahendra, S.; Lyon, D.Y.; Brunet, L.; Liga, M.V.; Li, D.; Alvarez, P.J. Antimicrobial nanomaterials for water disinfection and microbial control: Potential applications and implications. Water Res. 2008, 42, 4591-4602. [CrossRef] [PubMed]

4. Appendini, P.; Hotchkiss, J.H. Review of antimicrobial food packaging. Innov. Food Sci. Emerg. Technol. 2002, 3, 113-126. [CrossRef]

5. Yang, W.J.; Neoh, K.-G.; Kang, E.-T.; Teo, S.L.-M.; Rittschof, D. Polymer brush coatings for combating marine biofouling. Prog. Polym. Sci. 2014, 39, 1017-1042. [CrossRef]

6. Cao, Z.; Sun, Y. Polymeric n-halamine latex emulsions for use in antimicrobial paints. ACS Appl. Mater. Interfaces 2009, 1, 494-504. [CrossRef] [PubMed]

7. Siedenbiedel, F.; Tiller, J.C. Antimicrobial polymers in solution and on surfaces: Overview and functional principles. Polymers 2012, 4, 46-71. [CrossRef]

8. McDonnell, G.; Russell, A.D. Antiseptics and disinfectants: Activity, action, and resistance. Clin. Microbiol. Rev. 1999, 12, 147-179. [PubMed]

9. Bridier, A.; Briandet, R.; Thomas, V.; Dubois-Brissonnet, F. Resistance of bacterial biofilms to disinfectants: A review. Biofouling 2011, 27, 1017-1032. [CrossRef] [PubMed]

10. Tiller, J.C. Antimicrobial surfaces. In Bioactive Surfaces; Börner, H., Lutz, J., Eds.; Springer: Berlin/Heidelberg, Germany, 2010; pp. 193-217.

11. Kenawyel, R.; Worley, S.D.; Broughton, R. The chemistry and applications of antimicrobial polymers: A state-of-the-art review. Biomacromolecules 2007, 8, 1359-1384. [CrossRef] [PubMed]

12. Tashiro, T. Antibacterial and bacterium adsorbing macromolecules. Macromol. Mater. Eng. 2001, $286,63-87$. [CrossRef]

13. Ikeda, T.; Tazuke, S.; Watanabe, M. Interaction of biologically active molecules with phospholipid membranes. Biochim. Biophys. Acta (BBA) Biomembr. 1983, 735, 380-386. [CrossRef]

14. Sarapas, J.M.; Backlund, C.M.; deRonde, B.M.; Minter, L.M.; Tew, G.N. Romp- and raft-based guanidinium-containing polymers as scaffolds for protein mimic synthesis. Chemistry 2017, 23, 6858-6863. [CrossRef] [PubMed]

15. Kenawy, E.-R.; Imam Abdel-Hay, F.; Abou El-Magd, A.; Mahmoud, Y. Synthesis and antimicrobial activity of some polymers derived from modified amino polyacrylamide by reacting it with benzoate esters and benzaldehyde derivatives. J. Appl. Polym. Sci. 2006, 99, 2428-2437. [CrossRef]

16. Gratzl, G.; Paulik, C.; Hild, S.; Guggenbichler, J.P.; Lackner, M. Antimicrobial activity of poly(acrylic acid) block copolymers. Mater. Sci. Eng. C Mater. Biol. Appl. 2014, 38, 94-100. [CrossRef] [PubMed]

17. Kenawy, E.-R.; Abdel-Hay, F.I.; El-Shanshoury, A.E.-R.R.; El-Newehy, M.H. Biologically active polymers. V. Synthesis and antimicrobial activity of modified poly(glycidyl methacrylate-co-2-hydroxyethyl methacrylate) derivatives with quaternary ammonium and phosphonium salts. J. Polym. Sci. Part A Polym. Chem. 2002, 40, 2384-2393. [CrossRef]

18. Li, G.; Shen, J.; Zhu, Y. Study of pyridinium-type functional polymers. Ii. Antibacterial activity of soluble pyridinium-type polymers. J. Appl. Polym. Sci. 1998, 67, 1761-1768. [CrossRef]

19. Palermo, E.F.; Kuroda, K. Chemical structure of cationic groups in amphiphilic polymethacrylates modulates the antimicrobial and hemolytic activities. Biomacromolecules 2009, 10, 1416-1428. [CrossRef] [PubMed]

20. Ioannou, C.J.; Hanlon, G.W.; Denyer, S.P. Action of disinfectant quaternary ammonium compounds against staphylococcus aureus. Antimicrob. Agents Chemother. 2007, 51, 296-306. [CrossRef] [PubMed]

21. Jennings, M.C.; Minbiole, K.P.; Wuest, W.M. Quaternary ammonium compounds: An antimicrobial mainstay and platform for innovation to address bacterial resistance. ACS Infect. Dis. 2015, 1, 288-303. [CrossRef] [PubMed]

22. Zasloff, M. Antimicrobial peptides of multicellular organisms. Nature 2002, 415, 389-395. [CrossRef] [PubMed] 
23. Brogden, K.A. Antimicrobial peptides: Pore formers or metabolic inhibitors in bacteria? Nat. Rev. Microbiol. 2005, 3, 238-250. [CrossRef] [PubMed]

24. Timofeeva, L.; Kleshcheva, N. Antimicrobial polymers: Mechanism of action, factors of activity, and applications. Appl. Microbiol. Biotechnol. 2011, 89, 475-492. [CrossRef] [PubMed]

25. Ikeda, T.; Hirayama, H.; Yamaguchi, H.; Tazuke, S.; Watanabe, M. Polycationic biocides with pendant active groups: Molecular weight dependence of antibacterial activity. Antimicrob. Agents Chemother. 1986, 30, 132-136. [CrossRef] [PubMed]

26. Kanazawa, A.; Ikeda, T.; Endo, T. Polymeric phosphonium salts as a novel class of cationic biocides. Ii. Effects of counter anion and molecular weight on antibacterial activity of polymeric phosphonium salts. J. Polym. Sci. Part A Polym. Chem. 1993, 31, 1441-1447. [CrossRef]

27. Lienkamp, K.; Tew, G.N. Synthetic mimics of antimicrobial peptides-A versatile ring-opening metathesis polymerization based platform for the synthesis of selective antibacterial and cell-penetrating polymers. Chemistry 2009, 15, 11784-11800. [CrossRef] [PubMed]

28. Locock, K.E.; Michl, T.D.; Valentin, J.D.; Vasilev, K.; Hayball, J.D.; Qu, Y.; Traven, A.; Griesser, H.J.; Meagher, L.; Haeussler, M. Guanylated polymethacrylates: A class of potent antimicrobial polymers with low hemolytic activity. Biomacromolecules 2013, 14, 4021-4031. [CrossRef] [PubMed]

29. Pasquier, N.; Keul, H.; Heine, E.; Moeller, M.; Angelov, B.; Linser, S.; Willumeit, R. Amphiphilic branched polymers as antimicrobial agents. Macromol. Biosci. 2008, 8, 903-915. [CrossRef] [PubMed]

30. He, Y.; Heine, E.; Keusgen, N.; Keul, H.; Moller, M. Synthesis and characterization of amphiphilic monodisperse compounds and poly(ethylene imine)s: Influence of their microstructures on the antimicrobial properties. Biomacromolecules 2012, 13, 612-623. [CrossRef] [PubMed]

31. Xu, X.; Xiao, H.; Ziaee, Z.; Wang, H.; Guan, Y.; Zheng, A. Novel comb-like ionenes with aliphatic side chains: Synthesis and antimicrobial properties. J. Mater. Sci. 2012, 48, 1162-1171. [CrossRef]

32. Chen, C.Z.; Beck-Tan, N.C.; Dhurjati, P.; van Dyk, T.K.; LaRossa, R.A.; Cooper, S.L. Quaternary ammonium functionalized poly(propylene imine) dendrimers as effective antimicrobials: Structure-activity studies. Biomacromolecules 2000, 1, 473-480. [CrossRef] [PubMed]

33. Keul, H.; Möller, M. Synthesis and degradation of biomedical materials based on linear and star shaped polyglycidols. J. Polym. Sci. Part A Polym. Chem. 2009, 47, 3209-3231. [CrossRef]

34. Thomas, A.; Müller, S.S.; Frey, H. Beyond poly(ethylene glycol): Linear polyglycerol as a multifunctional polyether for biomedical and pharmaceutical applications. Biomacromolecules 2014, 15, 1935-1954. [CrossRef] [PubMed]

35. Kainthan, R.K.; Janzen, J.; Levin, E.; Devine, D.V.; Brooks, D.E. Biocompatibility testing of branched and linear polyglycidol. Biomacromolecules 2006, 7, 703-709. [CrossRef] [PubMed]

36. Frey, H.; Haag, R. Dendritic polyglycerol: A new versatile biocompatible material. Rev. Mol. Biotechnol. 2002, 90, 257-267. [CrossRef]

37. Fitton, A.O.; Hill, J.; Jane, D.E.; Millar, R. Synthesis of simple oxetanes carrying reactive 2-substituents. Synthesis 1987, 1140-1142. [CrossRef]

38. Hans, M.; Gasteier, P.; Keul, H.; Moeller, M. Ring-opening polymerization of $\varepsilon$-caprolactone by means of mono- and multifunctional initiators: Comparison of chemical and enzymatic catalysis. Macromolecules 2006, 39, 3184-3193. [CrossRef]

39. Deutsches Institut für Normung. Medical Microbiology-Susceptibility Testing of Microbial Pathogens to Antimicrobial Agents_Part 7: Determination of the Minimum Bactericidal Concentration (MBC) with the Method of Microbouillondilution; Deutsches Institut für Normung: Berlin, Germany, 2009.

40. Wang, Y.; Xu, J.; Zhang, Y.; Yan, H.; Liu, K. Antimicrobial and hemolytic activities of copolymers with cationic and hydrophobic groups: A comparison of block and random copolymers. Macromol. Biosci. 2011, 11, 1499-1504. [CrossRef] [PubMed]

41. Marlier, J.F.; O'Leary, M.H. Carbon kinetic isotope effects on the hydrolysis of aryl carbonates. J. Am. Chem. Soc. 1990, 112, 5996-5998. [CrossRef]

42. Marquardt, F.; Mommer, S.; Lange, J.; Jeschenko, P.; Keul, H.; Möller, M. Homoserine lactone as a structural key element for the synthesis of multifunctional polymers. Polymers 2017, 9, 130. [CrossRef]

43. Lienkamp, K.; Kumar, K.N.; Som, A.; Nusslein, K.; Tew, G.N. “Doubly selective” antimicrobial polymers: How do they differentiate between bacteria? Chemistry 2009, 15, 11710-11714. [CrossRef] [PubMed] 
44. Chattopadhyay, S.; Heine, E.T.; Keul, H.; Möller, M. Multifunctional poly(vinyl amine)s bearing azetidinium groups: One pot preparation in water and antimicrobial properties. Macromol. Biosci. 2014, 14, 1116-1124. [CrossRef] [PubMed]

45. Ganewatta, M.S.; Tang, C. Controlling macromolecular structures towards effective antimicrobial polymers. Polymer 2015, 63, A1-A29. [CrossRef] 